Research Paper

\title{
Upregulation of ATP Binding Cassette Subfamily C Member 5 facilitates Prostate Cancer progression and Enzalutamide resistance via the CDK1-mediated AR Ser81 Phosphorylation Pathway
}

\author{
Guangjie Ji\#, Shiming He\#, Cong Huang, Yanqing Gong ${ }^{\bowtie}$, Xuesong $\mathrm{Li}^{\bowtie}$ and Liqun Zhou ${ }^{\bowtie}$ \\ Institute of Urology, Peking University. Department of Urology, Peking University First Hospital. National Urological Cancer Center of China, Beijing, China. \\ \#These authors contributed equally to this work. \\ $\triangle$ Corresponding authors: Yanqing Gong, Institute of Urology, Peking University. Department of Urology, Peking University First Hospital. National \\ Urological Cancer Center of China, Beijing, China. Phone: +86 13488658965; E-mail: yqgong@bjmu.edu.cn; Xuesong Li, Institute of Urology, Peking University. \\ Department of Urology, Peking University First Hospital. National Urological Cancer Center of China, Beijing, China. Phone: +86 15801399116; E-mail: \\ pineneedle@sina.com; Liqun Zhou, Institute of Urology, Peking University. Department of Urology, Peking University First Hospital. National Urological \\ Cancer Center of China, Beijing, China. Phone: +86 13601239241; E-mail: zhoulqmail@sina.com.
}

(c) The author(s). This is an open access article distributed under the terms of the Creative Commons Attribution License (https://creativecommons.org/licenses/by/4.0/). See http://ivyspring.com/terms for full terms and conditions.

Received: 2021.02.19; Accepted: 2021.03.26; Published: 2021.04.12

\begin{abstract}
The treatment of advanced prostate cancer, castration-resistant prostate cancer, remains challenging. The mechanisms of action of ATP binding cassette subfamily $C$ member 5 (ABCC5) in prostate cancer and its relationship with drug resistance are still unclear. Expression and prognostic analyses of $A B C C 5$ were performed through bioinformatic methods and immunohistochemistry analyses in multiple public databases as well as in our own prostate cancer cohort. The biological function of $A B C C 5$ in prostate cancer cells was evaluated by in vitro and in vivo cell proliferation and migration and invasion assays. The regulation of CDK1 by $A B C C 5$ was determined via RT-qPCR, western blots, and immunofluorescence. ABCC5 was significantly overexpressed in prostate cancer and positively associated with unfavorable clinicopathological features and prognosis. Upregulation of $A B C C 5$ could enhance the cell proliferation, migration, and invasion of prostate cancer in vitro and in vivo. Mechanistically, $A B C C 5$ exerts a protumor effect by binding to and inhibiting the protein degradation of $C D K 1$, which promotes the phosphorylation of AR at Ser81 by CDK1 and activates the transcriptional activity of AR on target genes. Moreover, the addition of a CDK1 inhibitor or knockdown of CDK1 significantly improved the efficacy of enzalutamide on prostate cancer cells. The ABCC5-CDK1-AR regulatory pathway could be a potential therapeutic target for advanced prostate cancer, especially castration-resistant prostate cancer (CRPC), to enhance the therapeutic effect of enzalutamide.
\end{abstract}

Key words: Castration-resistant prostate cancer; ABCC5; CDK1; AR; enzalutamide

\section{Introduction}

Prostate cancer has become one of the most prevalent malignancies affecting the quality of life of older men worldwide [1,2]. Although there are a variety of treatments for prostate cancer, especially early-stage prostate cancer, effective treatments for advanced prostate cancer are lacking [3-6]. As a type of advanced prostate cancer with a poor prognosis, metastatic castration-resistant prostate cancer (mCRPC) has attracted increasing attention from scholars and physicians $[7,8]$. Particularly for patients with metastatic and castration-resistant prostate cancer, combination regimens including androgen deprivation therapy and radiotherapy and chemotherapy are not sufficiently effective [9-15]. The search for new and effective drug targets is important for improving the efficacy of prostate cancer treatment.

ATP binding cassette subfamily $\mathrm{C}$ member 5 (ABCC5), also called multidrug resistance-associated protein 5, has been reported to play an essential cancer-promoting role in various tumors [16-19]. Current studies on ABCC5 have mainly focused on 
the impact of its transmembrane protein transport function on the efficacy of cancer chemotherapy. ABCC5 (rs9823696) was identified as a new specific risk locus associated with esophageal adenocarcinoma [20]. Inhibition of the FOXM1-ABCC5 axis could increase the intracellular concentrations of paclitaxel and overcome paclitaxel chemoresistance in nasopharyngeal carcinoma [16]. Additionally, ABCC5 is downregulated by several microRNAs in multiple types of cancer [17, 21-23]. However, the concrete biological mechanisms of $\mathrm{ABCC} 5$ in tumor progression and drug resistance remain unclear, especially the downstream regulatory pathway of ABCC5.

In this study, we focused on the specific regulatory mechanism of ABCC5 in prostate cancer progression. We first demonstrated that ABCC5 was overexpressed in prostate cancer and that a high expression level of ABCC5 resulted in aggressive clinicopathologic features and an unfavorable prognosis. ABCC5 promotes the proliferation, migration, and invasion of prostate cancer tumor cells in vitro and in vivo. Through bioinformatics analysis, we found that the possible downstream molecule of ABCC5 is CDK1 and experimentally verified that ABCC5 inhibits the degradation of CDK1 through its binding to CDK1, which in turn activates the downstream AR signaling pathway to exert protumor effects. Moreover, the addition of RO-3306, an inhibitor of CDK1, significantly inhibited tumor growth and enhanced enzalutamide sensitivity, providing a potential therapeutic target for clinical treatments.

\section{Materials}

\section{Bioinformatic data collection and mining}

To comprehensively evaluate the expression level of ABCC5 in prostate cancer samples, we downloaded the transcriptome data and clinical information of prostate cancer cohorts in The Cancer Genome Atlas (TCGA, https://portal.gdc.cancer. gov/) and Gene Expression Omnibus (GEO, https:/ / www.ncbi.nlm.nih.gov/geo/). The relationship between ABCC5 expression and immune cell infiltration in prostate cancer was explored in the TIMER (Tumor Immune Estimation Resource) website (http:// timer.cistrome.org/) [24-26].

\section{Weighted-gene correlation network analysis (WGCNA)}

Weighted-gene correlation network analysis is a systems biology method that uses gene expression data to build a scale-free network [27]. First, we extracted the expression data of TCGA-PRAD samples to implement WGCNA. All the genes expressing NA according to the expression profile were removed. Two outlier samples were removed based on the clustering distance after calculating each gene's variance per sample and selecting genes with a standard deviation higher than zero. Next, we used the $\mathrm{R}$ software package WGCNA to construct a weighted coexpression network. To ensure that the network is a scale-free network, we chose a soft threshold of $\beta=6$. We converted the expression matrix into the adjacency matrix and then transformed the adjacency matrix into a topological matrix (TOM). Based on TOM, we used the averagelinkage hierarchical clustering method to cluster genes according to the standard of the mixed dynamic shear tree and set the minimum number of genes in network modules to 30. After using the dynamic shear method to determine the gene modules, we calculated the feature vector values (eigengenes) of each module in turn and then performed cluster analysis on the modules, merging the modules that were closer to each other into a new module and setting the height to 0.25 . A total of 18 modules were obtained, where the gray module is a collection of genes that cannot be aggregated into other modules. According to the feature vectors of each module, we calculated the gene trait significance $(\mathrm{GtS})$ between these modules and ABCC5 expression to measure the degree of relation between the genes and scores. GtS $=0$ indicates that the genes are not related to the phenotype.

\section{Patient selection}

We collected clinical information and formalinfixed paraffin-embedded samples from a total of 149 prostate cancer patients who underwent radical prostatectomy without preoperative therapy at Peking University First Hospital from January 2012 to December 2014. Our study was approved by the Biomedical Research Ethics Committee of Peking University First Hospital. All patients in this study signed an informed consent form.

\section{Cell culture and transfection}

The C4-2 and VCaP cell lines were obtained from the Institute of Cell Research, Chinese Academy of Science, Shanghai, China. Cell lines were cultured in RPMI 1640 (Corning, USA) medium supplemented with $10 \%$ fetal bovine serum (FBS) in $37^{\circ} \mathrm{C}$ incubators with $5 \% \mathrm{CO}_{2}$. We designed shRNA sequences for ABCC5 and CDK1 and then inserted them into the pLKO.1 vector. The details of the shRNA sequences for $\mathrm{ABCC} 5$ and $\mathrm{CDK} 1$ are shown in Supplementary Table S1. The coding DNA sequence of ABCC5 was produced by PCR and inserted into the PLVX vector 
with an N-terminal S-tag label. Lentivirus packaging was completed in HEK-293T cells with a three-vector system of interest vector, viral packaging vector (psPAX2), and viral envelope vector (pMD2G) at a 4:3:1 ratio. Finally, prostate cancer cells were infected with lentivirus and selected with puromycin (2 $\mu \mathrm{g} / \mathrm{mL})$ and hygromycin $(10 \mu \mathrm{g} / \mathrm{mL})$.

\section{Generation of enzalutamide-resistant (ENZ-R) cell lines}

ENZ-R cells were generated by culturing C4-2 and 22Rv1 cells under $10 \mu \mathrm{M}$ enzalutamide for six months before the experiment. After generation, ENZ-R cells were maintained in media with $10 \mu \mathrm{M}$ enzalutamide.

\section{RNA extraction and real-time quantitative PCR}

Total RNA was extracted from prostate cancer cells by using TRIzol reagent (Invitrogen; Thermo Fisher Scientific, Inc.) and reverse-transcribed with an RT-PCR kit (TransGENE, Beijing, China). Quantitative PCR was conducted in the ABI Prism 7000 fluorescent quantitative PCR system (Applied Biosystems, Foster City, CA, USA) according to the manufacturer's instructions. The expression level was normalized to that of TUBA, and the sequences of all the primers in this study are shown in Supplementary Table S2.

\section{Immunohistochemistry (IHC)}

All paraffin-embedded tissues were cut into 5 $\mu \mathrm{m}$ sections and placed on slides. After the paraffin wax was removed, the tissue was brought to a boil in $10 \mathrm{mM}$ sodium citrate buffer, $\mathrm{pH}$ 6.0, for ten minutes for antigen unmasking. Then, $3 \% \mathrm{H} 2 \mathrm{O} 2$ was added to the surface of the tissues to inactivate endogenous peroxidases for 20 minutes. Subsequently, 10\% goat serum was used to reduce nonspecific binding for 1 hour at room temperature. The tissue was incubated with anti-ABCC5 or anti-CDK1 primary antibody at $4^{\circ} \mathrm{C}$ overnight and then processed with staining reagent (PV-9000, ZSGB-Bio, China) according to the manufacturer's instructions. Each slide was scored as negative, weak, moderate, and strong based on the staining frequency and intensity by two independent pathologists.

\section{Western blot analysis}

Total cellular protein was extracted using $1 \%$ NP-40 containing $1 \mathrm{mM}$ phenylmethylsulfonyl fluoride (PMSF) and quantified through a bicinchoninic acid assay (Sigma, USA). The protein samples were separated in SDS-PAGE gels and transferred to polyvinylidene difluoride membranes. The protein bands were visualized with an
ImmobilonTM Western kit (Millipore, Billerica, MA) under the Syngene G: BOX imaging system (Frederick, USA). All information about the primary antibodies in this study is shown in Supplementary Table S3.

\section{Immunofluorescence}

Cancer cells were placed on glass slides for 24 hours before the experiment. The cells were fixed with 4\% paraformaldehyde, washed with PBS for 15 minutes, and then permeabilized with $0.5 \%$ Triton X-100. Subsequently, the cells were incubated with the primary antibodies at room temperature for 2 hours.

\section{Immunoprecipitation}

After treatment with $10 \mu \mathrm{M}$ MG132 for $24 \mathrm{~h}$, cellular protein was extracted with $1 \% \mathrm{NP}-40$ and 1 mM PMSF and quantified using a bicinchoninic acid assay. S-tagged beads or target primary antibody were added to whole cell lysates and incubated at $4{ }^{\circ} \mathrm{C}$ overnight. After washing and discarding the beads, we collected the supernatant liquid for further immunoblot analyses.

\section{S-tag pulldown assay and mass spectrometry analysis}

Protein lysates were prepared by homogenizing the cells in 1\% NP40 buffer containing PMSF and a proteinase inhibitor cocktail (Roche). Protein lysates were incubated with S-tagged beads (Novagen) at 4 ${ }^{\circ} \mathrm{C}$ for $2 \mathrm{~h}$. After centrifugation and three washes with $1 \%$ NP-40 buffer, the precipitated complex was boiled with protein loading buffer and separated by SDS-PAGE. The gel was stained with a silver stain kit (Beyotime). The precipitated complex was then analyzed by mass spectrometry. Mass spectrometry analysis was performed using an AB SCIEX MALDI TOF-TOF 5800 Analyzer.

\section{Wound healing assay}

After seeding the prostate cancer cells in 6-well plates for 24 hours, the cells were scratched with a 200 $\mu$ sterile pipette. The wound was photographed with a microscope (Leica DM IL, Leica Microsystems, Germany) equipped with a digital camera (Leica DFC300FX) 24, 48, 72, and 96 hours later.

\section{MTS cell proliferation assay}

A total of 2000 cells/well were seeded in a 96-well plate and cultured for 6 days. During this time period, cell proliferation was measured every day. After addition of $20 \%$ MTS reagent (Abcam, USA) diluted in PBS and incubation for 4 hours in standard culture conditions, we measured the absorbance at $490 \mathrm{~nm}$ to evaluate the cell proliferation. 
Table 1. Correlation of $A B C C 5$ protein expression and clinicopathological characteristics in IUPU-PRAD

\begin{tabular}{|c|c|c|c|}
\hline \multirow[t]{2}{*}{ Clinicopathological features } & \multicolumn{2}{|c|}{$\mathrm{ABCC} 5$} & \multirow[t]{2}{*}{$p$-value } \\
\hline & Low & High & \\
\hline Diagnosed age & & & 0.92 \\
\hline$\leq 60$ & 11 & 34 & \\
\hline$>60$ & 26 & 78 & \\
\hline Pathological T stage & & & 0.05 \\
\hline $\mathrm{T} 1-\mathrm{T} 2$ & 23 & 49 & \\
\hline T3-T4 & 14 & 63 & \\
\hline Gleason score & & & 0.001 \\
\hline $\mathrm{GS} \leq 7$ & 37 & 73 & \\
\hline GS $>7$ & 0 & 39 & \\
\hline
\end{tabular}

\section{Colony formation assay}

A total of 1000 cells/well were seeded in a 6-well plate and cultured for 2 weeks. Then, after discarding the medium, we fixed and stained the cancer cells with $0.5 \%$ crystal violet methanol solution for 1 hour. The samples were dried by airing, and the 6-well plates were scanned.

\section{Transwell migration and invasion assay}

In the Transwell migration assay, 10000 cells were plated in the upper chamber of a Transwell apparatus (24-well insert, $8 \mu \mathrm{m}$ pore size, Corning) containing $200 \mu \mathrm{l}$ of serum-free RPMI-1640. The lower chamber was filled with $700 \mu \mathrm{L}$ of RPMI-1640 containing $10 \%$ fetal bovine serum. After 24 hours, the adherent cells on the underside were stained with $0.5 \%$ crystal violet in methanol for 30 minutes.

In the Transwell Matrigel invasion assay, 1000 cells in a Transwell apparatus (24-well insert, $8 \mu \mathrm{m}$ pore size, Corning) were coated with Matrigel (diluted 1:8 with PBS, product number 354234, Corning, Inc., NY, USA) for the invasion assay. The culture conditions were the same as those described in the Transwell migration assay. After 24 hours, the adherent cells on the underside were stained with $0.5 \%$ crystal violet in methanol for 30 minutes. The cells on the underside were photographed with a microscope (Leica DM IL, Leica Microsystems) equipped with a digital camera (Leica DFC300FX) and counted.

\section{In vivo tumorigenicity assay}

A total of $1 \times 10^{7} \mathrm{VCaP}$ cells with upregulated ABCC5 levels were subcutaneously injected into the flanks of 4 week-old male BALB/c immunodeficient nude mice (eight mice per group). Tumor volumes were measured once every week for 4 weeks. Then, the tumors were removed, and their sizes and weights were determined. The animal study was approved by the Institutional Animal Experiment Committee of Peking University First Hospital.

\section{Statistical analyses}

All statistical tests were performed using $R$ version 3.6, including Student's $t$ test, the chi-square test, and the nonparametric test. The results are shown as the mean \pm SEM. The Kaplan-Meier method with the log-rank test was applied to calculate survival benefits. Differences with $\mathrm{p}$ less than 0.05 were considered statistically significant $\left({ }^{*} \mathrm{p}<0.05,{ }^{* *} \mathrm{p}\right.$ $<0.01$ and $\left.{ }^{* * *} \mathrm{p}<0.001\right)$.

\section{Results}

\section{ABCC5 is overexpressed in prostate cancer}

To fully and comprehensively explore the expression of ABCC5 in prostate cancer, RNA-seq data of prostate samples from TCGA, GEO, Memorial Sloan Kettering Cancer Center (MSKCC), and Chinese Prostate Cancer Genome and Epigenome Atlas (CPGEA) were analyzed. We analyzed the expression profiles of different prostate tissues from several databases and found that the expression levels of ABCC5 showed an obvious progressive increase in metastatic castration-resistant prostate cancer (mCRPC) compared with benign hyperplastic tissues and primary tumor foci (Figure $1 \mathrm{~A}-\mathrm{H}, \mathrm{p} \leq 0.002$ ). Moreover, ABCC5 mRNA expression was higher in prostate cancer tissues than in matched adjacent normal prostate tissues, as demonstrated in the CPGEA dataset (Figure 1I). Furthermore, to evaluate the expression of ABCC5 at the protein level, we analyzed a total of 149 prostate cancer samples from the Institute of Urology, Peking University prostate cancer dataset (IUPU-PRAD) by immunohistochemistry. Consistent with the transcriptome results, we also found that ABCC5 was strongly overexpressed in the tumor tissue at the protein level compared with normal prostate tissue (Figure 1I, Supplementary Figure 1).

\section{Upregulated ABCC5 correlates with poor prognosis}

To further explore the relationship between ABCC5 overexpression and clinicopathological characteristics, we compared ABCC5 expression in different pathological scores and tumor stages. As shown in Figure 2, we found that the expression level of ABCC5 was significantly higher in prostate cancer patients with higher GS scores from the CPGEA cohort $(p=0.039$, Figure 2A), MSKCC cohort $(p=$ 0.046 , Figure 2B), and TCGA-PRAD cohort $(p<0.001$, Figure 2C) than those with low scores. Similarly, ABCC5 expression was also highly associated with tumor stage $(p=0.006$, Figure 2D). Next, we evaluated the relationship between ABCC5 expression levels and the prognosis of prostate cancer 
patients in several prostate cancer cohorts, including CPGEA, MSKCC, and TCGA-PRAD. In CPGEA ( $\mathrm{p}=$ 0.0091), MSKCC $(p=0.041)$, and TCGA-PRAD $(p<$ 0.0001 ), we found shorter progression-free survival (RFS) times in prostate cancer patients with high ABCC5 expression than those with low expression (Figure 2E-G). Similarly, in TCGA-PRAD ( $p=0.026$ ) and MSKCC $(p=0.013)$, prostate cancer patients with high ABCC5 expression also had shorter overall survival times than those with low ABCC5 expression (Figure 2H-I). Furthermore, in multivariable Cox regression analysis (Table 2), we found that the expression of ABCC5 is independent predictor of RFS in TCGA-PRAD $(\mathrm{p}=0.025)$.

Table 2. Univariable and multivariable Cox regression analyses of progression-free survival in TCGA-PRAD cohort

\begin{tabular}{lllll}
\hline \multirow{2}{*}{ Characteristics } & \multicolumn{2}{l}{ Univariable analyses } & \multicolumn{2}{l}{ Multivariable analyses } \\
\cline { 2 - 5 } & HR $(95 \% \mathrm{CI})$ & $p$-value & HR $(95 \% \mathrm{CI})$ & $p$-value \\
\hline $\begin{array}{l}\text { Diagnosed age } \\
(>60 \text { vs. } \leq 60)\end{array}$ & $1.413(0.823,2.425)$ & 0.2 & $1.209(0.687,2.127)$ & 0.51 \\
$\begin{array}{l}\text { Gleason Score } \\
(>7 \text { vs. } \leq 7)\end{array}$ & $5.434(2.864,10.31)<0.001$ & $3.447(1.593,7.459)$ & $0.00167^{* *}$ \\
pT (T3-4 vs. T1-2) & $3.428(1.672,7.027)<0.001$ & $2.051(1.879,4.783)$ & $0.034^{*}$ \\
pN (N1 vs. N0) & $1.656(1.276,3.133)<0.001$ & $1.364(1.046,2.428)$ & $0.027^{*}$ \\
$\begin{array}{l}\text { ABCC5 expression } \\
\text { (high vs. low) }\end{array}$ & $4.069(2.18,7.596)<0.001$ & $2.281(1.106,4.706)$ & $0.025^{*}$ \\
\hline
\end{tabular}

TCGA-PRAD, The Cancer Genome Atlas Prostate Adenocarcinoma.
A

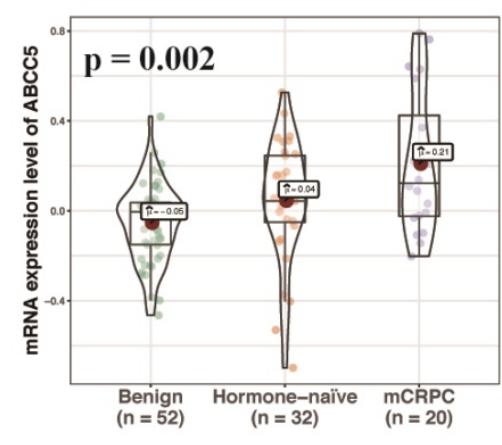

D

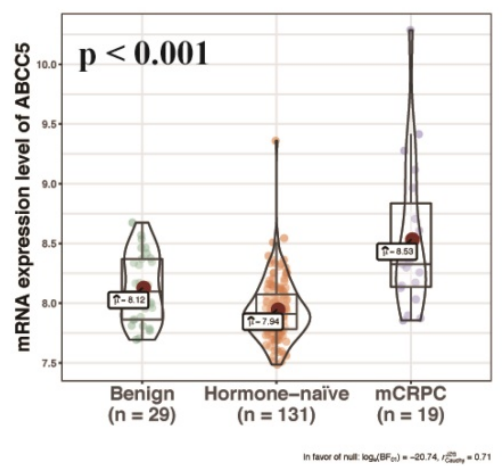

G

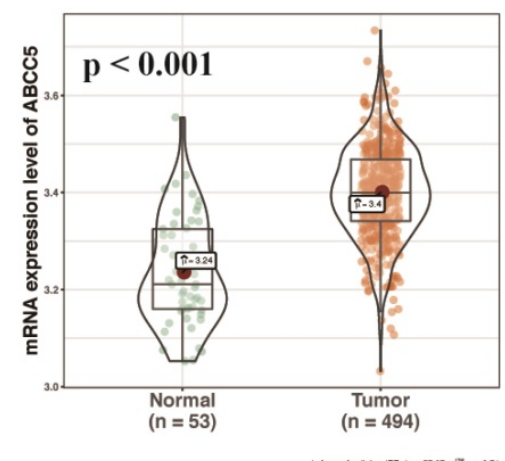

B
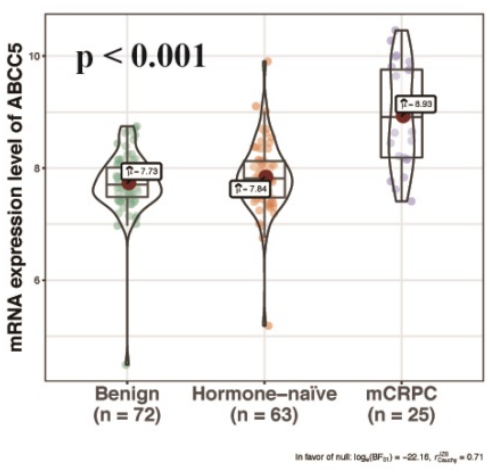

$\mathbf{E}$

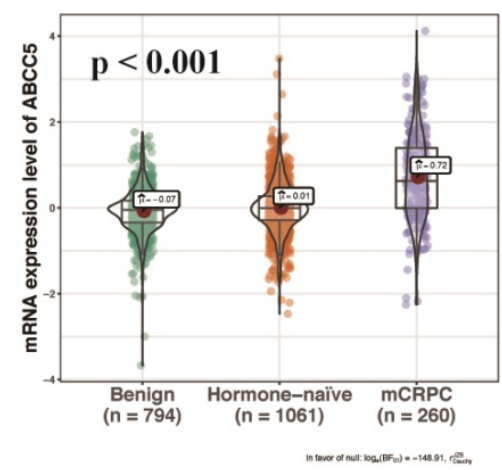

H

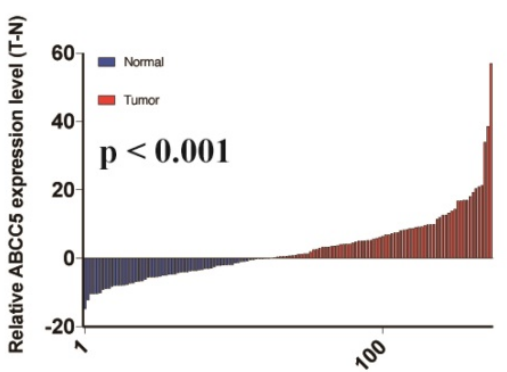

C

GSE8511

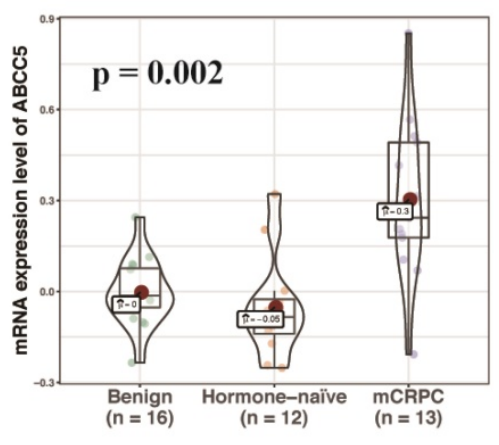

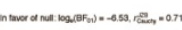

F MSKCC

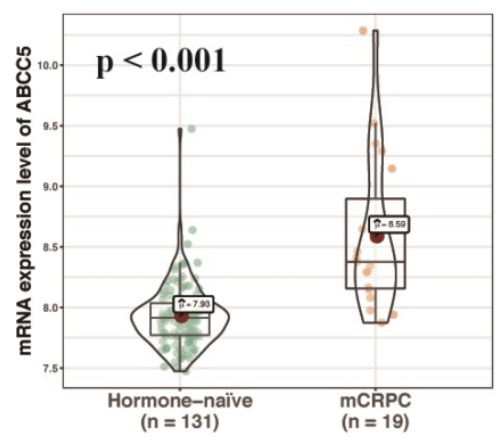

I

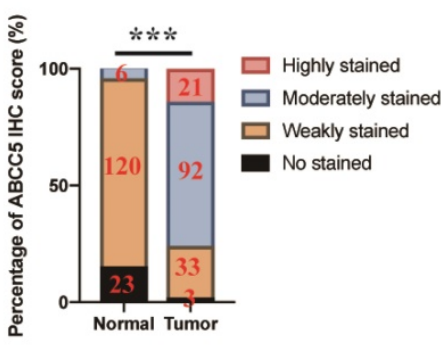

Figure 1. The expression of ABCC5 is upregulated in prostate cancer from different datasets. A. GSE6099, B. GSE6919, C. GSE8511, D. GSE21034, E. PCTA, F. MSKCC, G. TCGA-PRAD, H. CPGEA, I. IUPU-PRAD. PCTA, prostate cancer transcriptome atlas. MSKCC, Memorial Sloan Kettering Cancer Center prostate cancer cohort. TCGA-PRAD, The Cancer Genome Alta Prostate Adenocarcinoma. CPGEA, Chinese Prostate Cancer Genome and Epigenome Atlas. IUPU-PRAD, Institute of Urology, Peking University, Prostate Adenocarcinoma. ***P $<0.001$ 
A

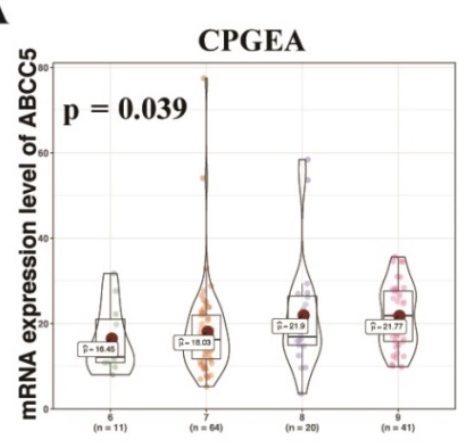

D

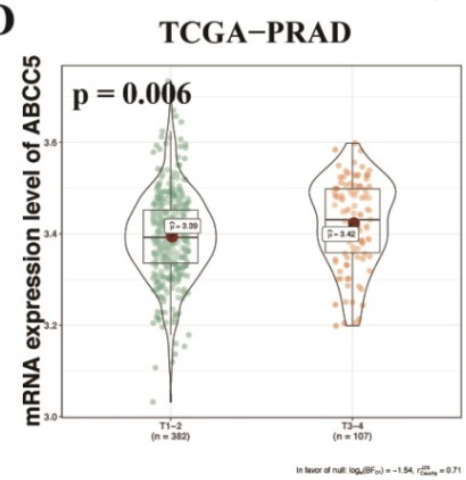

G
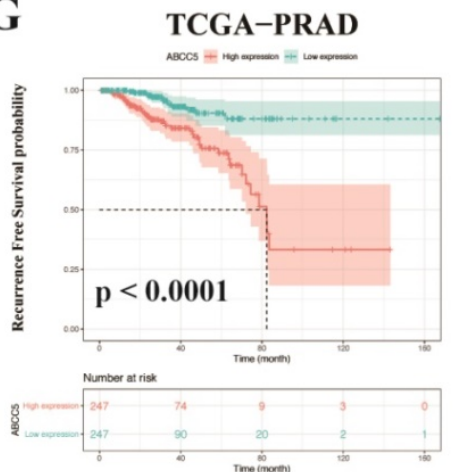

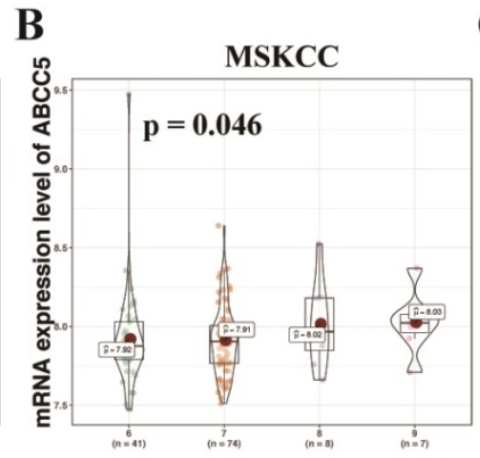

E

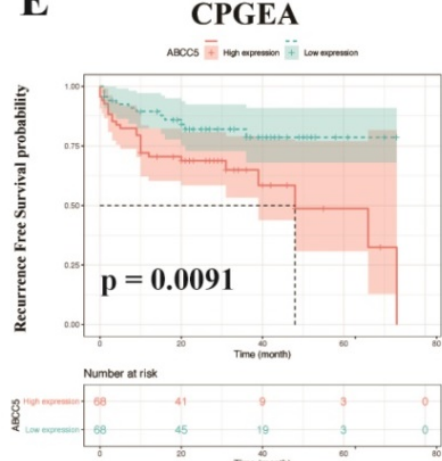

H

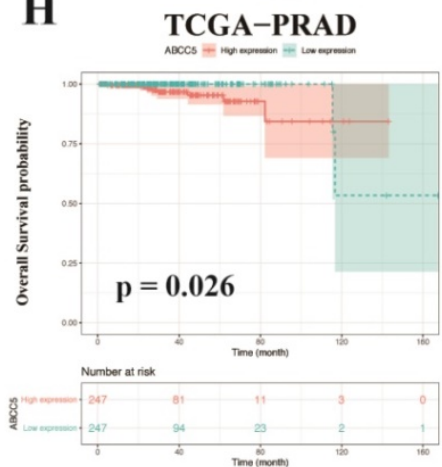

C

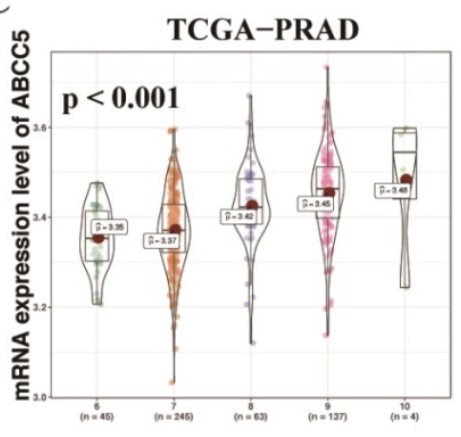

F
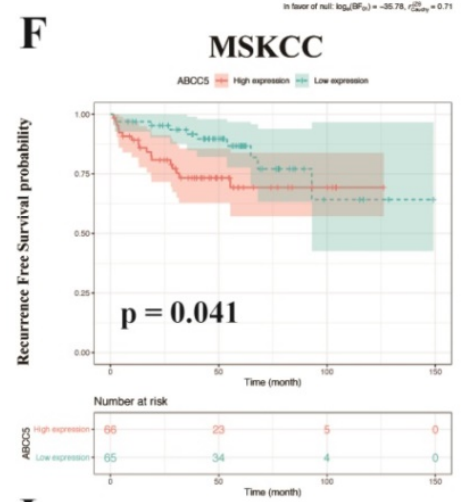

I

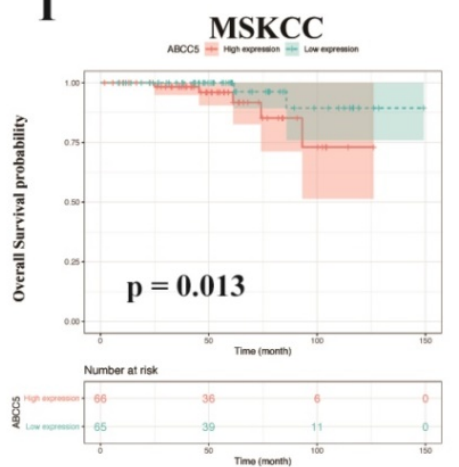

Figure 2. ABCC5 expression is associated with pathological features and prognosis of prostate cancer. A-C. Association of $A B C C 5$ expression with different $G S$ of prostate cancer in CPGEA (A), MSKCC (B), PCGA-PRAD (C). D. Association of ABCC5 expression with different pathological stages of prostate cancer in TCGA-PRAD. E-G. Recurrence survival curves of ABCC5 high and low expression groups in CPGEA (E), MSKCC (F), PCGA-PRAD (G). H-I. Overall survival curves of ABCC5 high and low expression groups in TCGA-PRAD (H), MSKCC (I). CPGEA, Chinese Prostate Cancer Genome and Epigenome Atlas. MSKCC, Memorial Sloan Kettering Cancer Center prostate cancer cohort. TCGA-PRAD, The Cancer Genome Alta Prostate Adenocarcinoma.

\section{ABCC5 facilitates the proliferation, migration and invasion of prostate cancer cells}

To further explore the effect of ABCC5 on prostate cancer, we knocked down and overexpressed ABCC5 in two prostate cancer cell lines, C4-2 and $\mathrm{VCaP}$, respectively (Figure $3 \mathrm{~A}-\mathrm{C}$ ), and performed a series of tumor cell functional assays. The results of MTS and clone formation assays showed that the proliferation of prostate cancer cells was significantly inhibited and enhanced by ABCC5 knockdown and overexpression, respectively (Figure 3D-E). Subsequently, we examined the effect of ABCC5 on the migration of prostate cancer cells, and the results of scratch and transwell migration assays showed that knockdown of ABCC5 significantly reduced the migration of prostate cancer cells, while overexpression of $\mathrm{ABCC} 5$ significantly enhanced the migration of prostate cancer cells (Figure 3F-H, Supplementary Figure 2A-B). Similarly, the invasive ability of prostate cancer cells was suppressed with the downregulation of $\mathrm{ABCC} 5$ expression and enhanced with the increase in ABCC5 expression (Figure 3I). The procarcinogenic role of ABCC5 in prostate cancer was also confirmed by the results of the rescue assay (Supplementary Figure 2C-D). To further validate the effect of ABCC5 on prostate cancer in animals, we chose to perform subcutaneous tumorigenesis experiments using ABCC5overexpressing $\mathrm{VCaP}$ cells injected into immunodeficient nude mice. We found that high expression of ABCC5 significantly accelerated the tumor growth of prostate cancer (Figure 3J). 
A

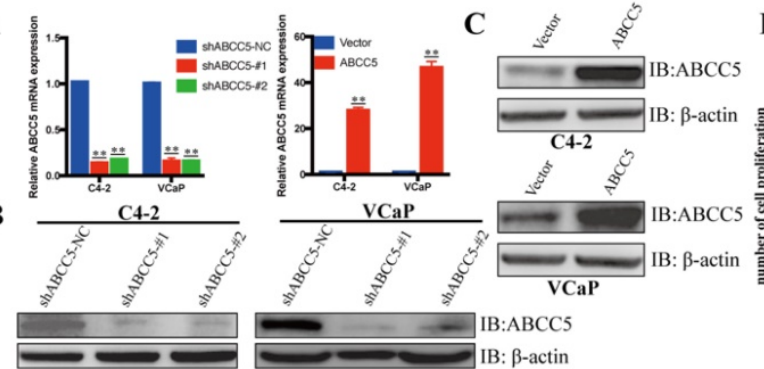

D
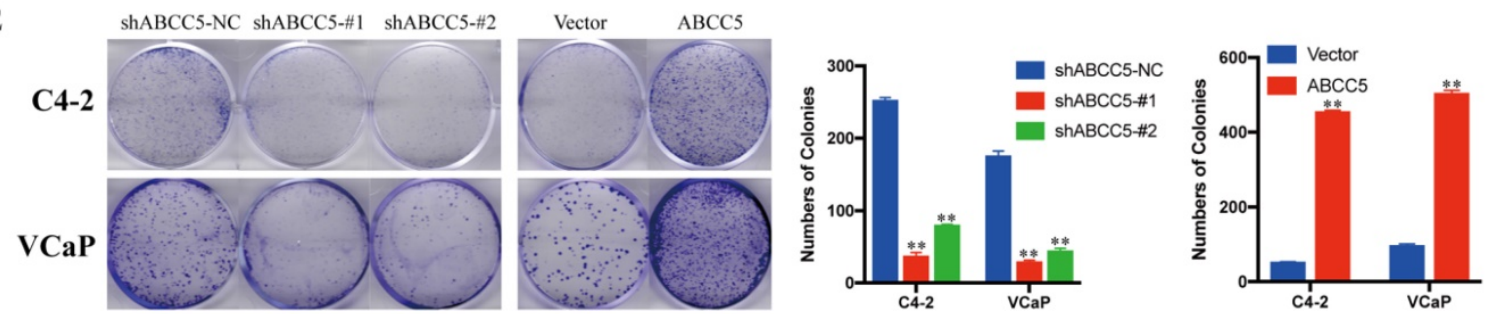

F

$\mathrm{Oh}$

shABCC5-NC shABCC5-\#1 shABCC5-\#2

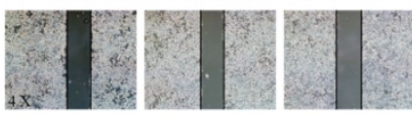

$24 \mathrm{~h}$

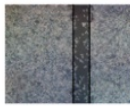

$72 \mathrm{~h}$
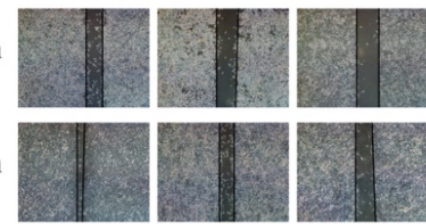

H

H

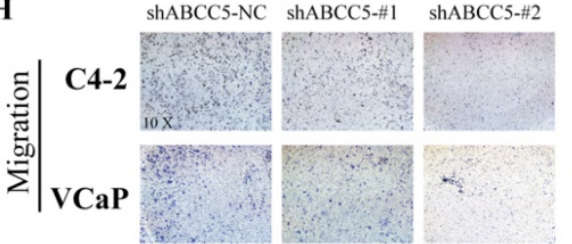

I

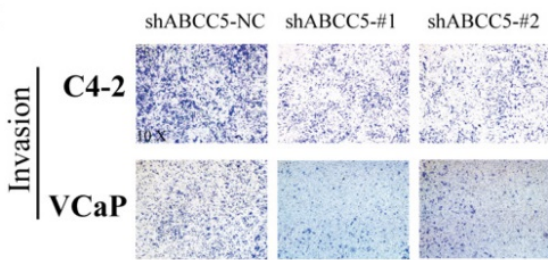

G
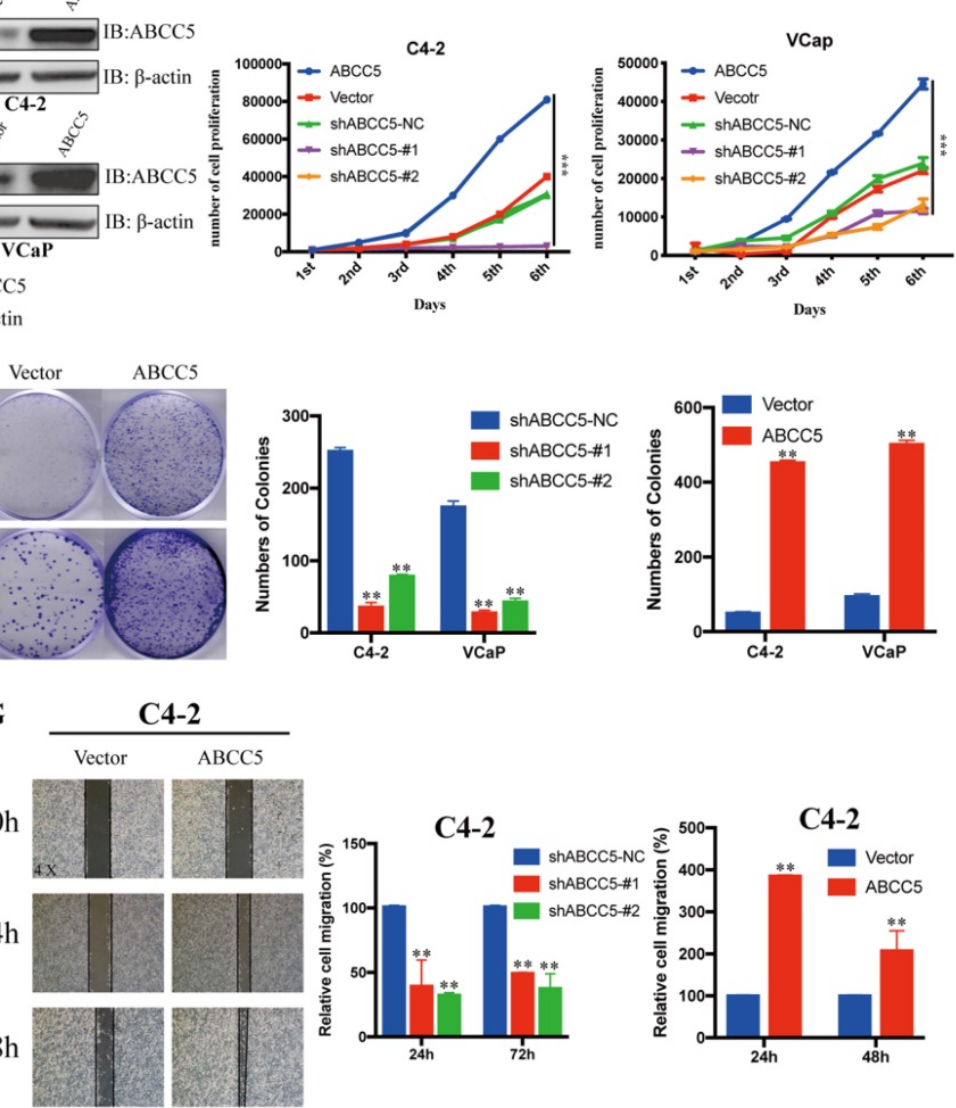

ector
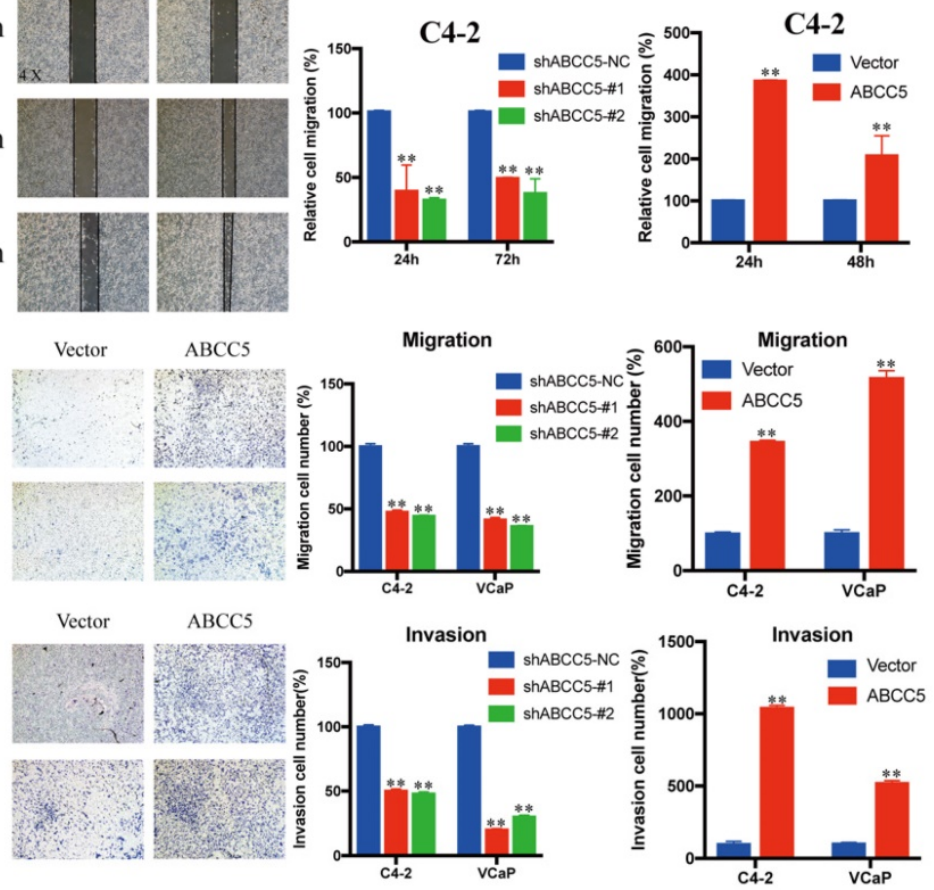

$\mathbf{J}$
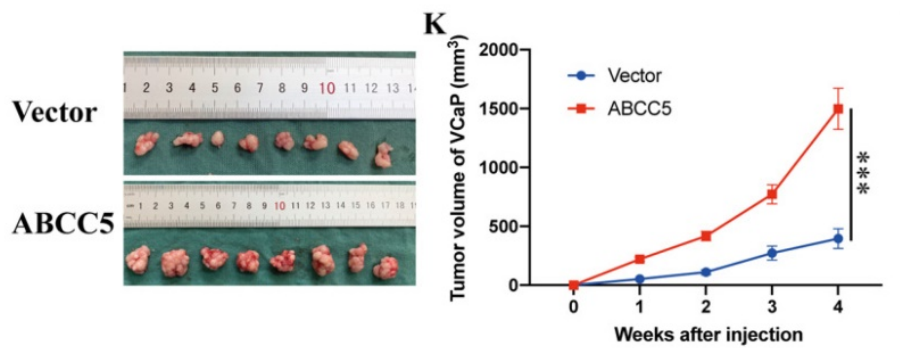

L

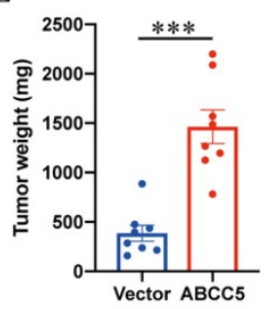

Figure 3. ABCC5 promotes the malignant progression of prostate cancer in vivo and in vitro. A-C. RT-qPCR and western blot analyses of prostate cancer cells infected with a lentivirus-mediated ABCC5-overexpressing vector or ABCC5 shRNAs. D. MTS cell proliferation assay of prostate cancer cells infected with a lentivirus-mediated $A B C C 5$-overexpressing vector or $A B C C 5$ shRNAs. E. Colony formation assay, Representative images of clone formation of shABCC5, $A B C C 5$ and control group in $C 4-2$ and VCaP cells (left). Quantification of colonies by the described cells (right). F-G. Wound healing assay, Representative images of wound-induced cell migration by the C4-2-shABCC5 (F), C4-2-ABCC5 (G) and control cells (4x, left). Quantification of migration by the described cells (right). H. Transwell migration assay, Representative images of transwell migration assay of $A B C C 5$-knockdown and $A B C C 5$-overexpressed and control cells (10x, left). Quantification of transwell migration by the described cells (right). I. Transwell invasion assay, Representative images of transwell invasion assay of ABCC5-knockdown and ABCC5-overexpressed and control cells (10x, left). Quantification of transwell invasion by the described cells (right). J-L. Subcutaneous tumorigenesis experiment in nude mice. Representative images of subcutaneous tumor of nude mice injected with $A B C C 5$-overexpressed and control cells. Eight mice in each group (J). Growth curve of subcutaneous tumor volume (K). Comparison of tumor weight at 4 th week between control and $\mathrm{ABCC} 5$-overexpressed groups $(\mathrm{L})$. ** $\mathrm{P}<0.01$, *** $\mathrm{P}<0.001$. 


\section{Downstream pathway prediction of ABCC5 combined with bioinformatics analysis}

To explore the specific mechanism of the cancer-promoting effect of ABCC5 in prostate cancer, we divided the prostate cancer patients in the TCGA-PRAD cohort into an ABCC5 high expression group and an $\mathrm{ABCC} 5$ low expression group according to the expression level of ABCC5. By comparing the differential expression of all genes in these two groups, we obtained a total of 11,676 genes with upregulated expression in the ABCC5 high expression group compared to the ABCC5 low expression group. We performed GO analysis of downstream pathways and GSEA of these differentially expressed genes and found that $\mathrm{ABCC} 5$ highly expressed in prostate cancer may act through multiple tumor-related pathways, including the PI3K-Akt and MAPK/ERK signaling pathway and E2F target genes (Figure 4A-B). Previous studies have reported a regulatory relationship between the PI3K-Akt and MAPK/ERK signaling pathways and CDK1 $[28,29]$. Immediately thereafter, we analyzed transcriptomic data from prostate cancer patients using the WGCNA approach and found that the pink module was highly correlated with ABCC5 expression levels $(R=0.39, p=4 e-19)$ (Figure 4C-E). Moreover, 387 genes in the pink module were significantly correlated with ABCC5 (Figure 4F). Using protein pulldown and mass spectrometry techniques, we then searched for proteins that might bind to $A B C C 5$ and in this way mediate the oncogenic effects of ABCC5 at the protein level. We identified 69 proteins that may bind to $\mathrm{ABCC} 5$ in the cell lines overexpressing ABCC5 compared to the controls, focusing on biochemical processes such as protein membrane localization and molecular functions such as ubiquitinated protein binding (Figure 4G). Finally, we assessed the intersection of the set of differentially expressed genes positively associated with ABCC5 expression, the full set of genes in the pink module that are highly associated with ABCC5, and the proteins identified by protein profiling that may interact with $\mathrm{ABCC} 5$ and identified a potential downstream molecule, CDK1, that may interact with ABCC5 (Figure 4H).

\section{ABCC5 binds CDK 1 and stabilizes the protein levels}

To further explore the regulatory relationship between $\mathrm{ABCC} 5$ and $\mathrm{CDK} 1$, we examined the mRNA and protein expression levels of CDK1 in the ABCC5 knockdown cell lines and the ABCC5 overexpression cell lines. We found that ABCC5 could stabilize the protein level of CDK1 without affecting mRNA expression (Figure 5A-C). Further, we proposed that ABCC5 could specifically bind and stabilize the protein level of CDK1. To fully test our hypothesis, we examined the protein expression levels of ABCC5 and CDK1 in our own prostate cancer cohort and revealed that the protein expression of CDK1 was highly positively correlated with the protein expression of ABCC5 (Figure 5D-E). Immediately afterward, we also confirmed that ABCC5 could bind to CDK1 using IP (Figure 5F, Supplementary Figure 3A). By laser confocal immunofluorescence, we verified that ABCC5 could indeed bind and upregulate CDK1; thus, does ABCC5 stabilize the protein level of CDK1 by inhibiting its protein degradation? We found that the half-life of CDK1 was prolonged and that ubiquitinated CDK1 was reduced after overexpression of ABCC5 (Figure 5H-J), suggesting that ABCC5 binds to CDK1 and inhibits the ubiquitination of CDK1, thus stabilizing the CDK1 protein levels. We also found that ABCC5 could activate the ERK1/2 and Akt signaling pathway (Figure 5K-L, Supplementary 3B, C), which confirmed the results of the previous signal pathway analyses.

\section{Inhibition of CDK1 blocked the cancer-promoting effect of ABCC5}

To verify whether CDK1 mediates the pro-oncogenic effect of $\mathrm{ABCC} 5$ in prostate cancer, we knocked down CDK1 again in prostate cancer cells overexpressing $\mathrm{ABCC} 5$ and performed a series of tumor phenotyping experiments. We found that inhibition of CDK1 expression was followed by a significant blockade of the ability of ABCC5 to proliferate and migrate in prostate cancer (Figure 6A, C, D). Then, we inhibited CDK1 kinase activity with the CDK1-specific inhibitor RO-3306 and found that the ability of highly expressed ABCC5 to promote tumor proliferation and migration was also inhibited when CDK1 kinase activity was inhibited (Figure 6B, C, D). Based on previous studies showing that CDK1 can specifically phosphorylate AR at serine 81 to activate AR transcriptional activity, we hypothesize that the pro-cancer effect of ABCC5 is mediated by stabilizing CDK1 to activate AR phosphorylation. Our experimental results also confirmed this point. After the upregulation of $\mathrm{ABCC} 5$ expression, not only was the protein level of CDK1 increased but also the level of AR phosphorylated at Ser81 was significantly upregulated, and the transcriptional activity of AR downstream target genes was significantly enhanced. Therefore, ABCC5 enhances the specific phosphorylation of AR at Ser81 by CDK1 through binding to CDK1 and inhibiting its protein degradation, which in turn stabilizes the protein level of CDK1 and activates the transcriptional activity of $\mathrm{AR}$ on downstream target genes to promote the malignant progression of prostate cancer. 
A

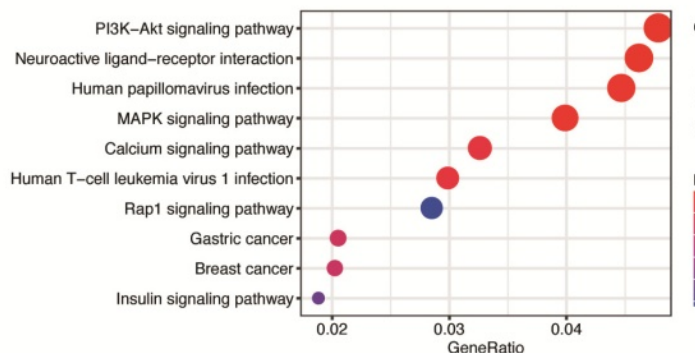

C

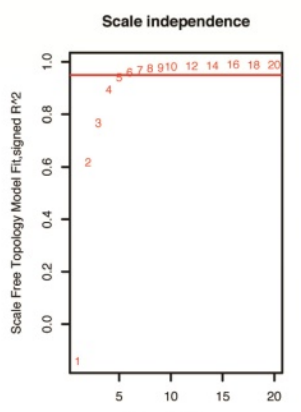

Solt Threshold (power)

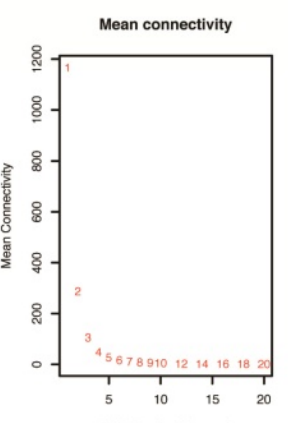

Soft Threshold (power)

E

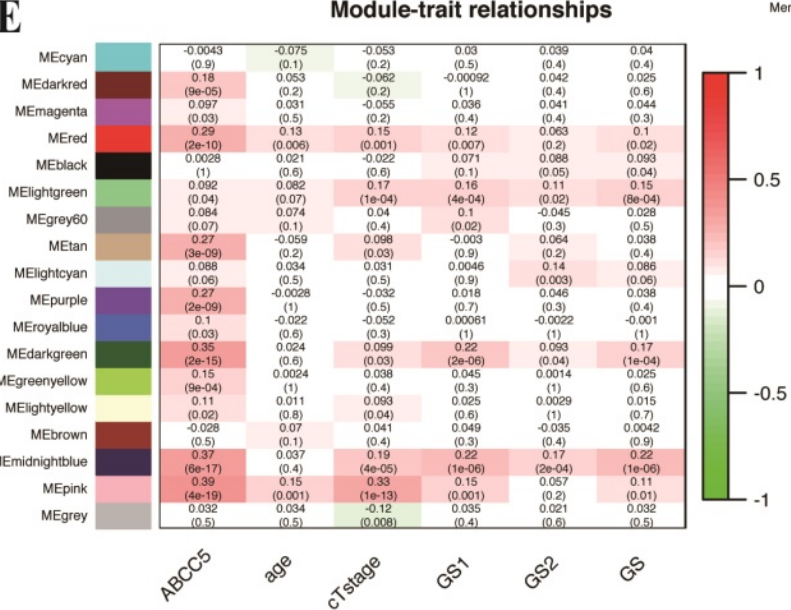

G

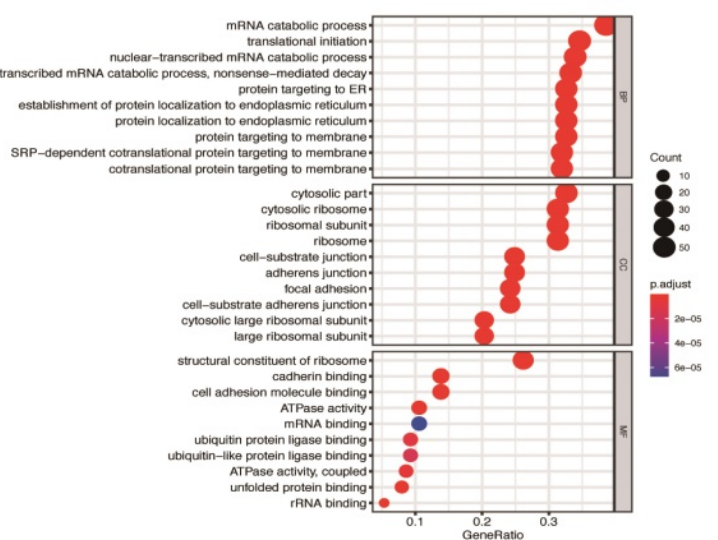

B

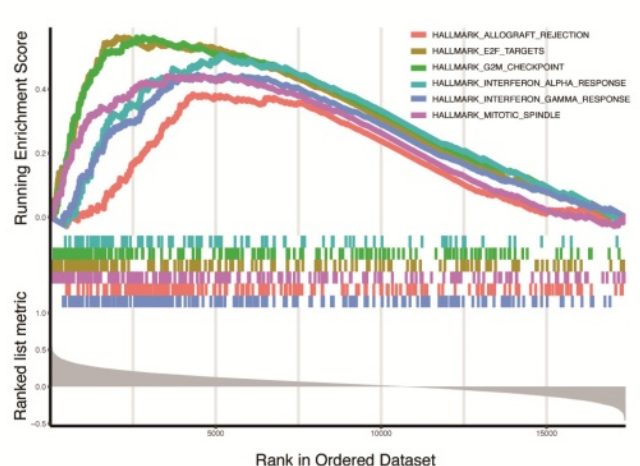

D

Cluster Dendrogram
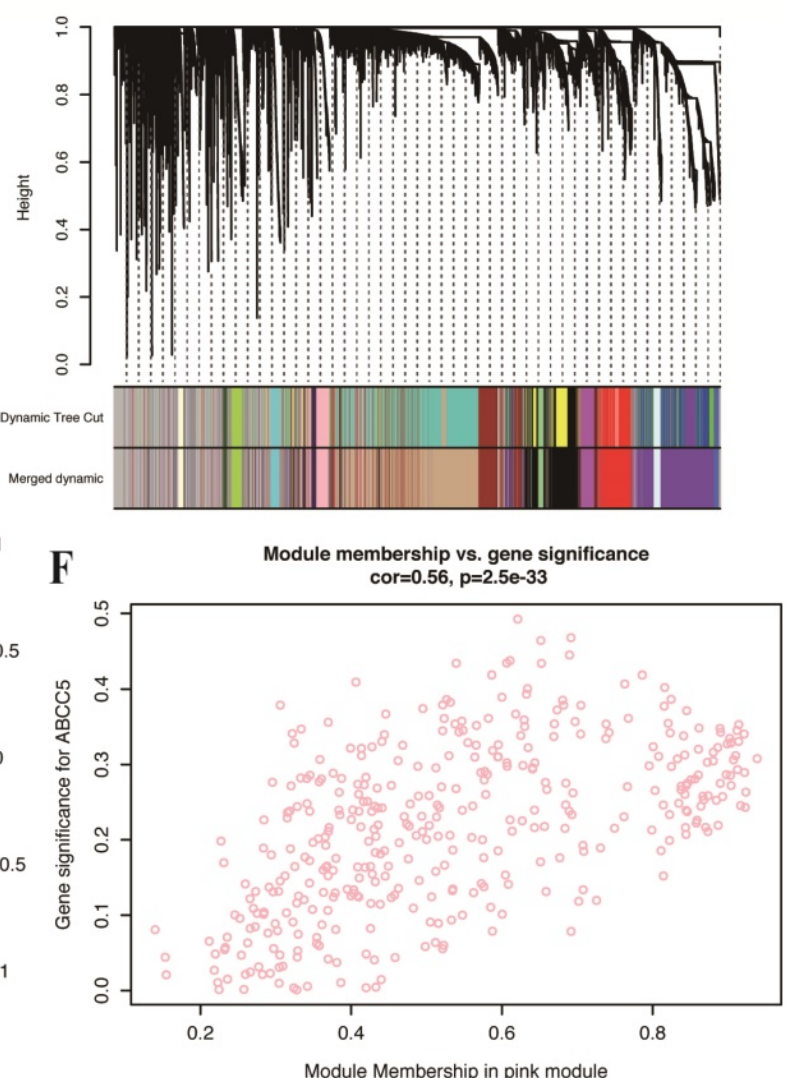

H

Genes positively associated

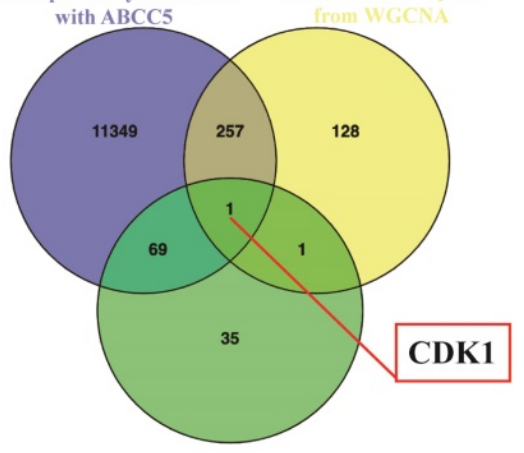

ABCC5 S-tag pull down assay

Figure 4. CDK1 may be the potential molecular in the downstream of ABCC5 in prostate cancer based on bioinformatic analyses. $A$. GO analysis of genes positively associated with ABCC5 in TCGA-PRAD. B. GSEA plot of genes positively associated with ABCC5 based on hallmarks gene sets. C. Optimizing soft threshold to make sure there is a scale-free network. D. cluster dendrogram of module construction. E. correlation between modules and ABCC5 expression and other clinicopathological features of prostate cancer. F. correlation between gene significance in module pink and ABCC5 expression. G. GO analysis of proteins in ABCC5 S-tag pull-down assay. H. The intersection of genes positively associated with $A B C C 5$, genes in module pink from WGCNA, and proteins of ABCC5 S-tag pull-down assay. GO, gene ontology. TCGA-PRAD, The Cancer Genome Alta Prostate Adenocarcinoma. WGCNA, Weighted-gene correlation network analysis. 
A
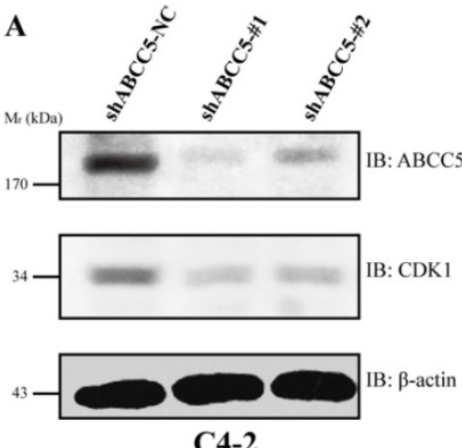

C4-2

D

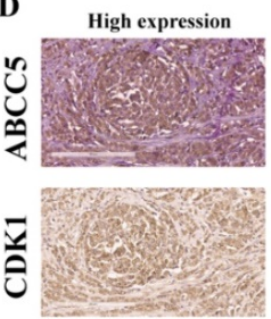

GS: $5+4$
B

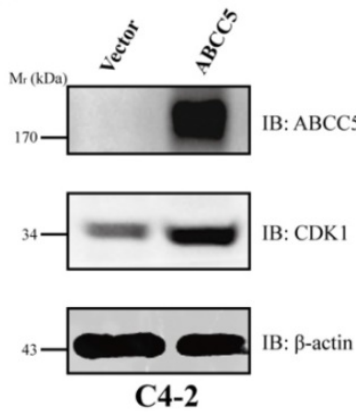

E Correlation between ABCC5 and CDK1 at protein level

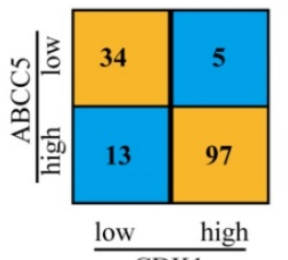

C

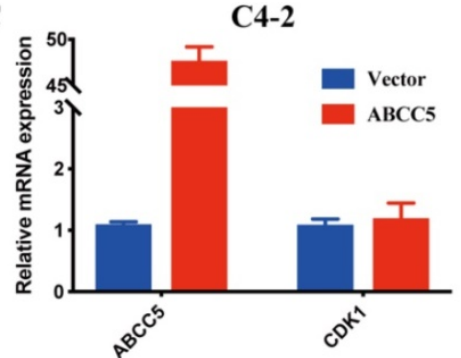

F
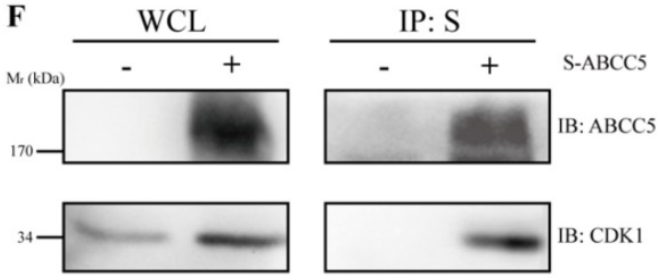

IB: $\beta$-actin
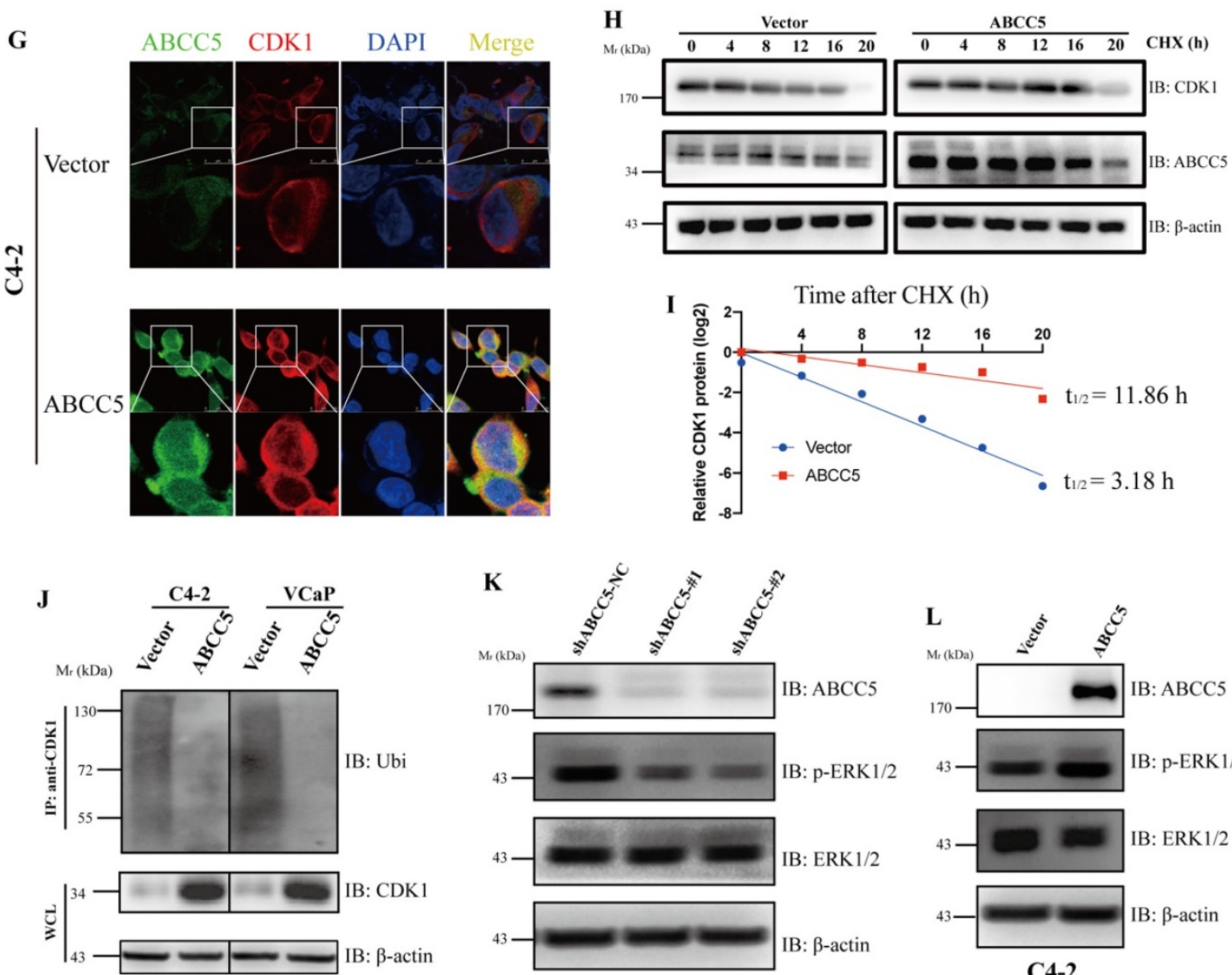

C4-2

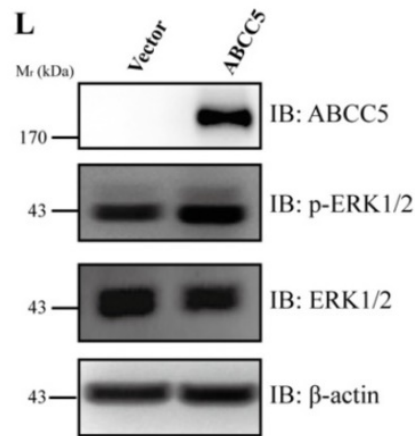

C4-2

Figure 5. ABCC5 upregulates CDK1 protein expression by binding to and inhibiting the ubiquitinated degradation of $C D K 1$. $A$. Knockdown of $A B C C 5$ reduced the protein expression of $C D K 1$ in the prostate cancer cell. B. Overexpressed ABCC5 increased the protein expression of $C D K 1$ in the prostate cancer cell. $C$. The mRNA expression of CDK1 in ABCC5-overexpressed and control cells. D. Representative images of ABCC5 and CDK1 protein expression in IUPU-PRAD cohort by IHC. Scale bar $200 \mu \mathrm{m}$. E. Correlation between $A B C C 5$ and $C D K 1$ at the protein level. $F$. The result of immunoprecipitation revealed that $A B C C 5$ binds to $C D K 1$ at the protein level ( $P$ $<0.01$ ). G. ABCC5 and CDK1 were co-localized by immunofluorescence. $\mathrm{H}-\mathrm{I}$. ABCC5 prolongs the half-life of $C D K 1$. Control and $A B C C 5$ overexpressing prostate cancer cells were treated with Cycloheximide $(\mathrm{CHX})(50 \mu \mathrm{M})$, and then the protein levels of $C D K 1$ were detected at different time periods. J. ABCC5 inhibits the ubiquitin proteasome-dependent degradation pathway of CDK1. Control and ABCC5 overexpressing prostate cancer cells were treated with the proteasome inhibitor MG132 ( $10 \mu \mathrm{M})$ for 24 hours, and then ubiquitinated CDK1 expression was examined. K-L. Knockdown and overexpression of ABCC5 can inhibit and activate the $\mathrm{P}-\mathrm{ERK} 1 / 2$ pathway in prostate cancer cells, respectively. 
A

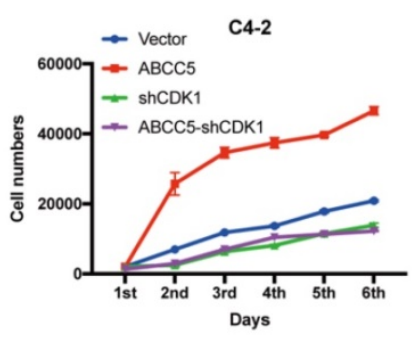

C

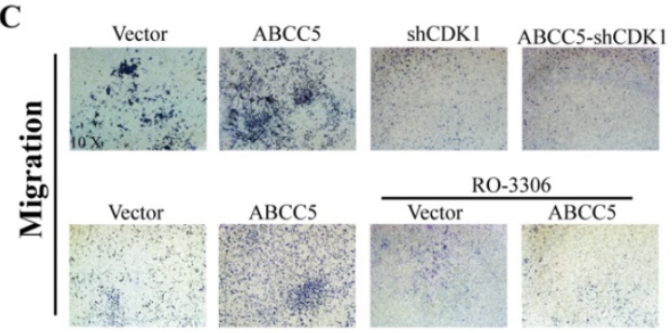

C4-2
B

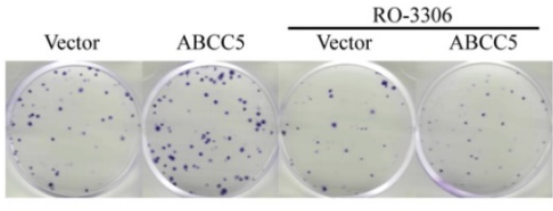

C4-2

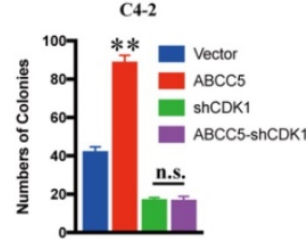

C4-2
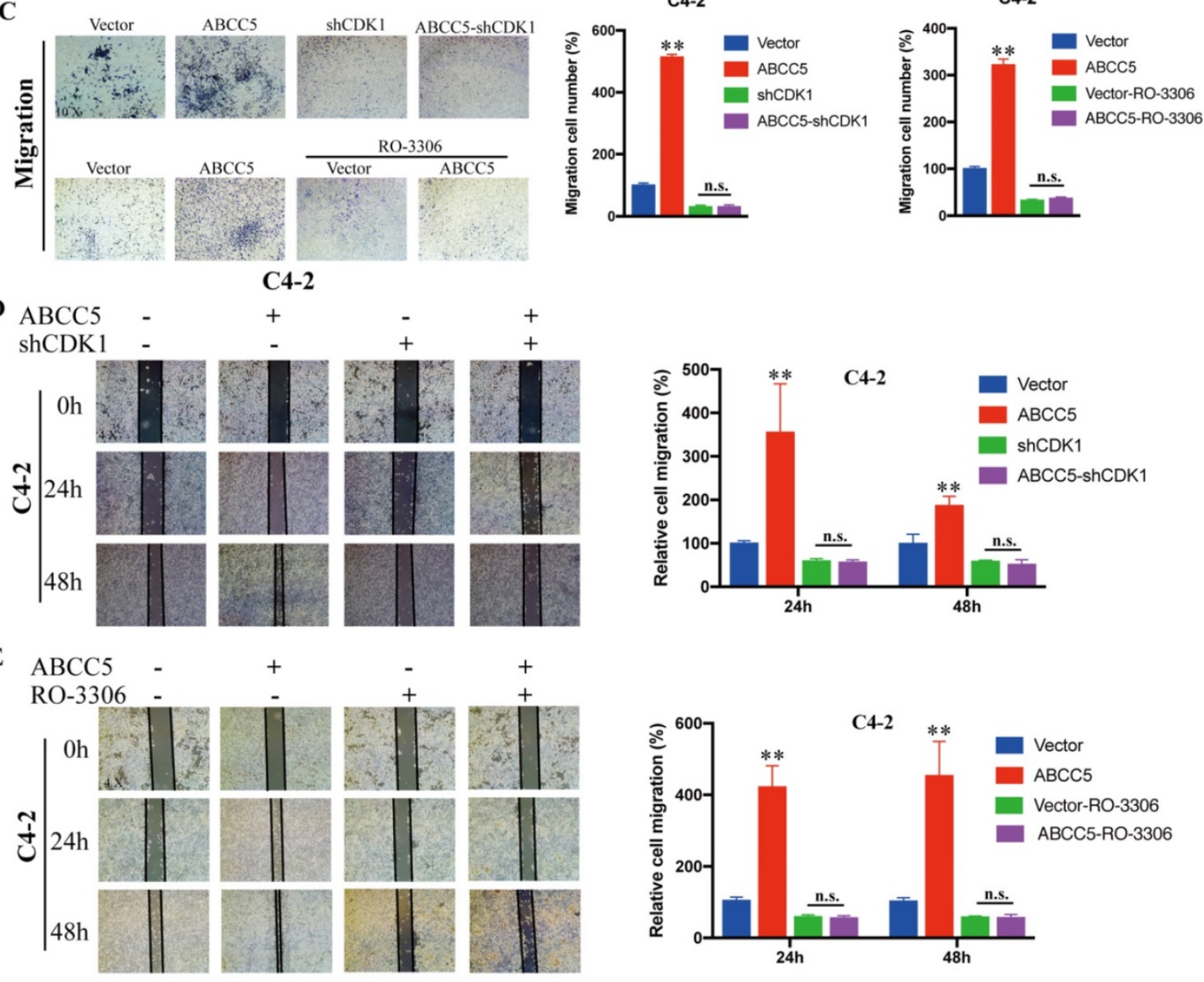

F

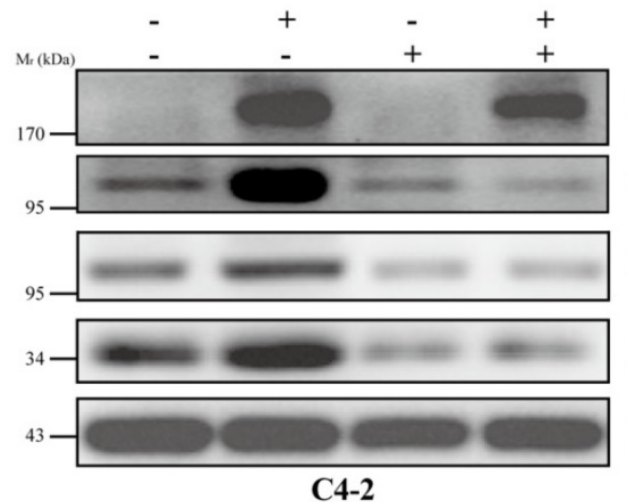
ABCC5 shCDK1
IB: $\mathrm{ABCC} 5$
IB: p-AR (Ser 81)
IB: AR
IB: CDK1
IB: $\beta$-actin

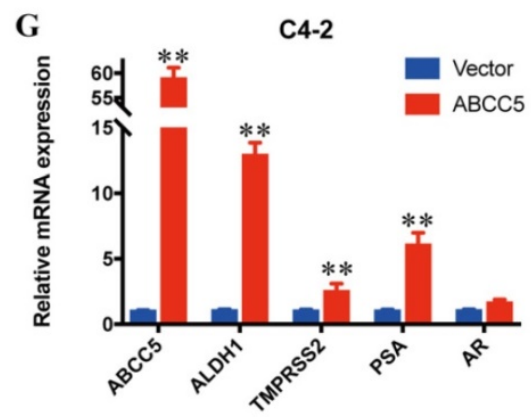

Figure 6. CDK1 mediates the cancer-promoting effect of $A B C C 5$ on prostate cancer. A. MTS cell proliferation assay. Cell growth curves of ABCC5 and ABCC5shCDK1 and control cells. B. Colony formation assay. Representative images of colonies of ABCC5 with or without RO-3306 treated cells (left). Quantification of the number of colonies by the described cells (right). C. Transwell migration assay. Representative images of ABCC5 and ABCC5-shCDK1 and control cells (10x, top), ABCC5 with or without RO-3306 treated cells (10x, bottom). Quantification of cell migration rate by the described cells (right). D-E. Wound healing assay. Representative images of wound-induced cell migration of $A B C C 5$-overexpressed and CDK1-inhibited and control cells (4x, left). Quantification of migration by the described cells (right). F. ABCC5 upregulates the protein level of $C D K 1$, which in turn promotes phosphorylation of the AR (ser81). G. ABCC5 upregulates the transcriptional activity of $A R$ on downstream target genes. **P $<0.01$. n.s., none of significance. ENZ., enzalutamide. 
A

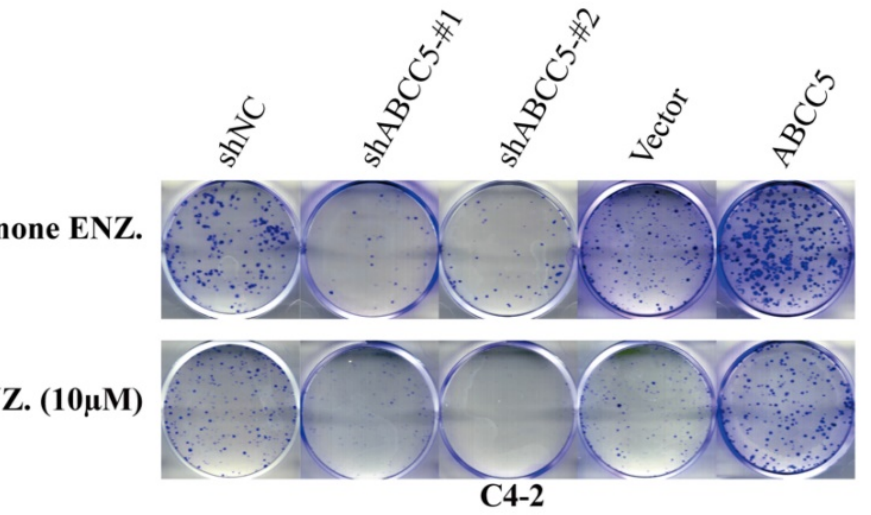

B

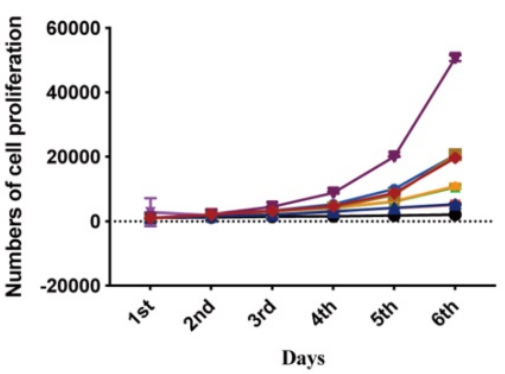

$\rightarrow$ shNC none ENZ

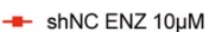

ঊ sh\#1 none ENZ

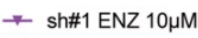

$\rightarrow-$ sh\#2 none ENZ

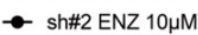

-- Vector none ENZ

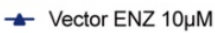

$\rightarrow$ ABCC5 none ENZ

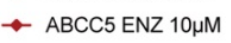

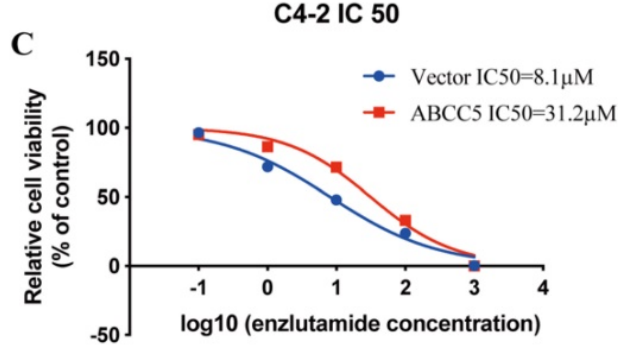

D

E

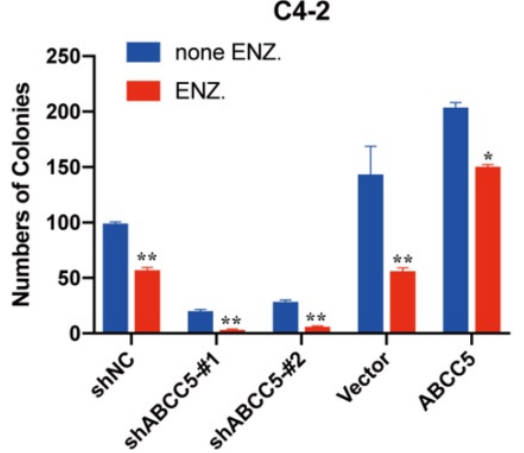

C4-2 IC 50

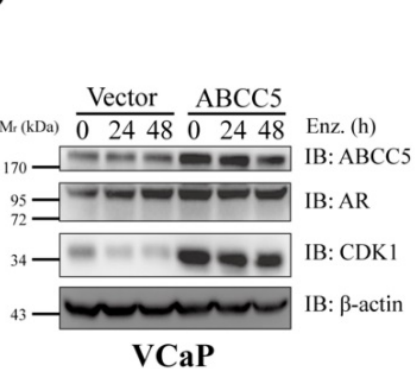

Mr(kDa) $\frac{\text { Vector }}{0} \quad \frac{\text { ABCC5 }}{248} \frac{2448 \text { Enz. (h) }}{0}$ $170-2-2=$ IB: ABCC5 95${ }_{34-\infty}=\mathbf{m}$ IB: CDK1 IB: $\beta$-actin C4-2

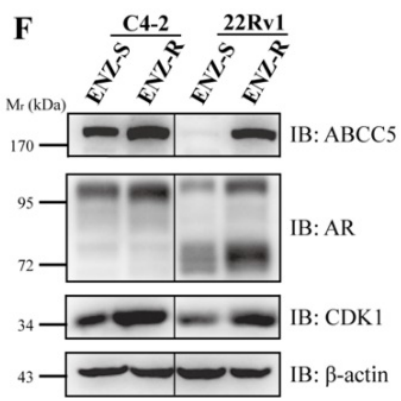

G
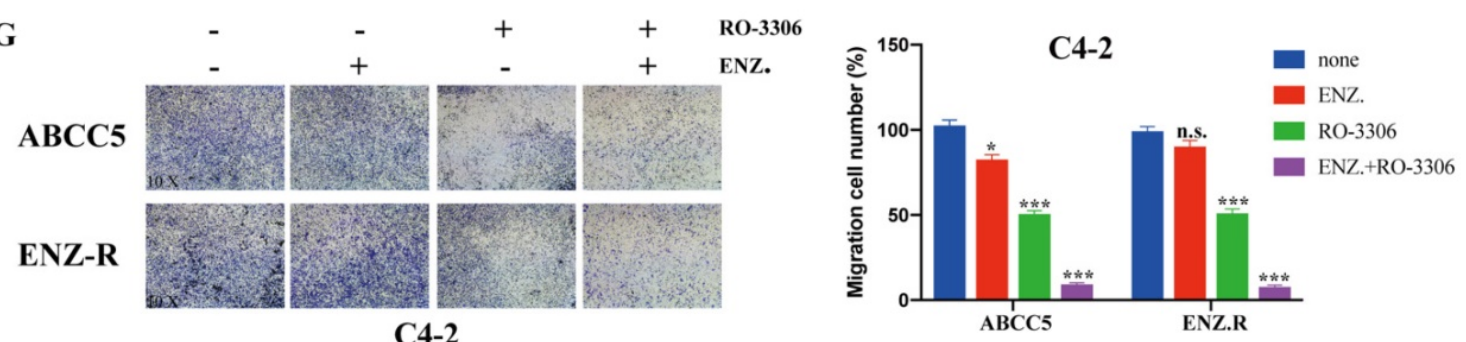

H
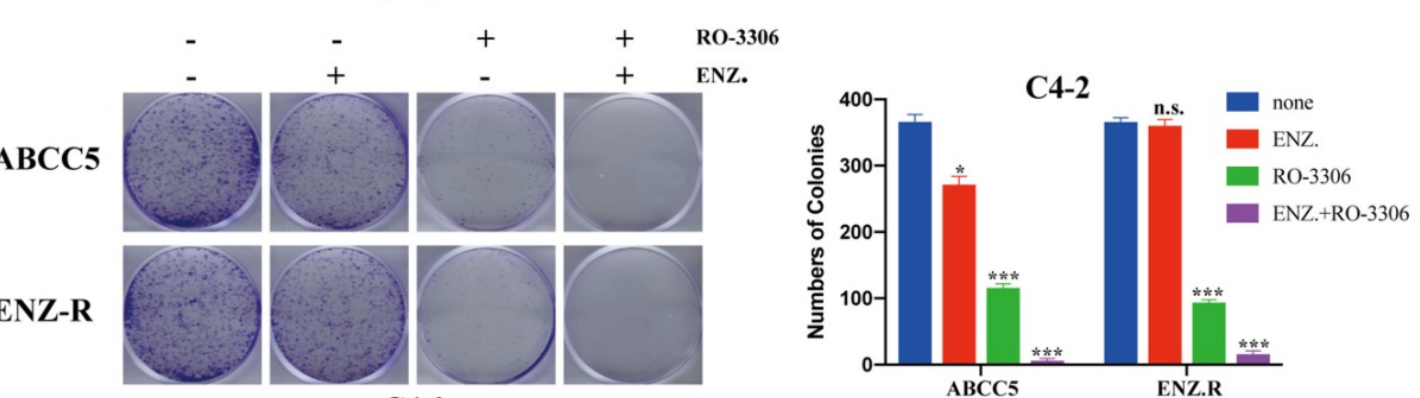

C4-2

Figure 7. Inhibition of CDK1 increases the sensitivity of prostate cancer cells to Enzalutamide. A. Colony formation assay. Representative images of $A B C C 5$-shRNA, ABCC5-overexpressed and control cells treated with or without enzalutamide (10 $\mu$ M) for 24 hours (left). Quantification of colony numbers by the described cells (right). B. MTS cell proliferation assay. Cell growth curves of ABCC5-shRNA, ABCC5-overexpressed and control cells treated with or without enzalutamide (10 $\mu M$ ). $C$. Cell viability curves of $A B C C 5$-overexpressed and control cell treated with different concentration enzalutamide (10 $\mu M)$. D-E. The protein expression in $A B C C 5$-overexpressed and control cells treated with enzalutamide $(10 \mu \mathrm{M})$ for 0,24 , and 48 hours in VCaP (D) and C4-2 (E) cell lines. F. ABCC5 is over-expressed in ENZ-R cells. G. Transwell migration assay. Representative images of transwell migration assay of ABCC5-overexpressed and ENZ-R cells treated with or without RO-3306 (1 $\mu$ M) and/or enzalutamide (10 $\mu M)(10 x$, left). Quantification of cell migration by the described cells (right). H. Colony formation assay. Representative images of colony formation of ABCC5-overexpressed and ENZ-R cells treated with or without RO-3306 (1 $\mu \mathrm{M})$ and/or enzalutamide $(10 \mu \mathrm{M})(10 \mathrm{x}$, left). Quantification of colonies number by the described cells (right). 


\section{CDK1 inhibitors enhance the sensitivity of prostate cancer cells to enzalutamide}

ABCC 5 has been highly associated with tumor cell resistance in previous studies [16, 19, 30-32]. Enzalutamide resistance in prostate cancer, especially in patients with castration-resistant prostate cancer, has become an important challenge in clinical treatment. We investigated whether the mechanism of ABCC5-CDK1 in prostate cancer is related to enzalutamide resistance. First, we found that upregulated $\mathrm{ABCC} 5$ reduced the sensitivity of CRPC cells to enzalutamide (Figure 7A-C). We treated CRPC cells overexpressing ABCC5 with enzalutamide for a short period of time and found that enzalutamide did not significantly alter the protein expression of ABCC5, CDK1 and AR (Figure 7D-E). However, significant upregulation of ABCC5, CDK1, and AR expression was found in ENZ-R cells, suggesting that the formation of enzalutamide resistance is associated with the ABCC-CDK1 pathway. Finally, we found that the combination of the CDK1 inhibitor RO-3306 and enzalutamide significantly increased the killing effect on CRPC cells (Figure 7G-H).

\section{Discussion}

In this study, we discovered the mechanism of ABCC5 in tumor development, namely, promoting malignant phenotypes such as proliferation and migration of prostate cancer by stabilizing CDK1 protein levels and activating ERK signaling pathways. At present, there are very few studies related to the role of $\mathrm{ABCC} 5$ in prostate cancer, and the conclusion that $\mathrm{ABCC} 5$ is highly expressed in prostate cancer and correlates with the malignancy of the tumor in the present study fills the gap in this field of research to some extent. Similarly, in 2006, Zhang et al. also found that ABCC5 expression was higher in prostate cancer than in normal paracarcinoma tissue [33]. Although Karatas and his colleagues used RT-qPCR to detect nine $A B C$ transporter proteins, including $A B C C 5$, in prostate cancer specimens after radical prostate cancer surgery and normal prostate tissue, they found that ABCC5 was not significantly different in the tumor and normal groups and was not associated with recurrence of prostate cancer [34]. However, in this study, by comparing ABCC5 mRNA expression levels in metastatic and primary prostate cancer foci from the TCGA prostate cancer database and several GEO prostate cancer databases, we showed that ABCC 5 was abnormally highly expressed in prostate cancer metastases and found a higher pathological Gleason score as well as a shorter biochemical recurrence-free survival in prostate cancers with high expression of ABCC5. This cancer-promoting effect of
ABCC5 was also validated in our own samples. This finding suggests that $\mathrm{ABCC} 5$ plays an important role in the development of prostate cancer, especially high-risk prostate cancer and that $\mathrm{ABCC} 5$ can serve as a prognostic biomarker for high-risk prostate cancer and provide a new target for prostate cancer diagnosis and treatment.

The existing studies on the downstream mechanisms of ABCC5 regulation in tumorigenesis and development are relatively few and mainly focus on the upstream regulatory mechanisms of ABCC5. Some scholars found that ABCC5 is the target of miRNA-210 and miRNA-516a-3p, and the deletion of these miRNAs upregulated the protein level of ABCC5, which in turn promoted tumor cell proliferation and migration, as well as the formation of chemotherapy resistance $[17,35]$. Our study mainly focused on exploring the downstream regulatory mechanisms of ABCC5 in prostate cancer progression. In this study, by protein profiling and immunoprecipitation techniques, ABCC5 was found to bind to CDK1 in the cytoplasm and inhibit the ubiquitination-mediated degradation of CDK1 to upregulate the protein level of CDK1, which in turn promotes the activation of AR phosphorylation by CDK1, thus exerting a protumor effect.

Many previous studies have shown that high expression of ABCC5 is closely associated with the development of drug resistance after tumor treatment $[16-19,22,23,30-32,36-39]$. ABCC5 is not only a signaling protein in many physiological processes via pumping cGMP out of cell, but also a drug transport pump confering multitype chemotherapy resistances. However, the substrate specificity of the ABCC5 transporter is still uncertain. Some research showed that $\mathrm{ABCC} 5$ was not associated with drug resistance to anthracycline antibiotics, mitoxantrone, Vinca alkaloids, paclitaxel, and cisplatin [40,41], while in later studies presented that ABCC5 could contribute to diminish anticancer efficacy of doxorubicin, 6-mercaptopurine (6-MP), 6-thioguanine (6-TG), 5-FU, paclitaxel, irinotecan, celecoxib, and 9-(2-phosphonylmethoxyethyl) adenine (PMEA) [42-44]. In fact, our results showed that high expression of ABCC5 reduced the sensitivity of prostate cancer cells to enzalutamide. The IC50 of enzalutamide in ABCC5overexpressing prostate cancer cells was $31.2 \mu \mathrm{M}$, which was significantly higher than that in the control group (Figure 7). Moreover, in enzalutamide-resistant prostate cancer cells, we found that the expression level of ABCC5 was upregulated compared to that in enzalutamide-sensitive tumor cells. This finding suggests that ABCC5 plays a vital role in the development of enzalutamide resistance in prostate cancer cells. Our previous study found that the 
immune microenvironment in metastatic and primary sites of prostate cancer is very different, suggesting whether ABCC5 plays a role in the tumor microenvironment. Then, we evaluated the relationship between the expression of ABCC5 and immune cell infiltration in prostate cancer based on the TCGA database (Supplementary Figure 4). The results showed that $A B C C 5$ expression is significantly associated with the infiltration of B cells, CD4+ T cells, macrophages, neutrophils, and dendritic cells in the tumor microenvironment. This finding suggests that the role of $\mathrm{ABCC} 5$ in prostate cancer is of interest.

CDK1 is known to play an essential role in the management of the cell cycle and tumor development. Previous studies have demonstrated that high expression of CDK1 facilitates the poor prognosis of prostate cancer [45-48]. In prostate cancer, the activation of CDK1 is linked with the development of CRPC and enzalutamide resistance via copy number loss of 17q22 [45]. CDK1 mediates AR Ser81 phosphorylation and stabilizes AR [49]. Moreover, androgen-independent AR activity is driven by CDK1-mediated AR Ser81 phosphorylation in CRPC [47]. Consistent with previous studies, our study found that ABCC5 binds to CDK1 and stably upregulates CDK1 protein levels by inhibiting its ubiquitination-mediated degradation, which in turn activates CDK1-mediated phosphorylation of AR Ser81, promoting the proliferation and invasive capacity of prostate cancer cells and the development of resistance to enzalutamide. The malignant proliferation and migration of prostate cancer caused by ABCC5 was significantly eliminated by inhibiting CDK1 activity. The combination of enzalutamide and RO-3306 significantly inhibited the growth of prostate cancer cells with high ABCC5 expression. This discovery will help provide valuable potential therapeutic targets for the clinical treatment of enzalutamide-resistant CRPC patients. Previous studies have found that in the cytoplasm, phosphorylated inactivated CDK1 is recognized and bound by 14-3-3 proteins [50]. In the present study, protein profiling results also revealed that ABCC5 may be present in 14-3-3 protein binding and scored significantly lower than the binding score of ABCC5 to $\mathrm{CDK} 1$. This point reinforces that $\mathrm{ABCC} 5$ plays a critical role in stabilizing CDK1.

Our study has certain shortcomings or limitations. First, this study did not determine which specific protein is the E3 ligase that mediates CDK1 ubiquitination in prostate cancer. Second, we did not clarify the respective protein sequences in the binding regions of $A B C C 5$ and $C D K 1$ in this study, which we will continue in future studies. Finally, the role of CDK1 phosphorylation in regulating ABCC5- mediated CDK1 protein stability has not been evaluated. We are also conducting research to address these as yet unidentified mechanisms.

In conclusion, we found that the expression of ABCC5 was higher in prostate cancer tissue than in normal tissue and that prostate cancer patients with high ABCC5 expression had a poorer prognosis. Both in vitro and in vivo experiments confirmed that overexpression of $\mathrm{ABCC} 5$ promotes the malignant progression of prostate cancer. Furthermore, ABCC5 stabilizes the protein level of CDK1 by binding to and inhibiting the ubiquitin proteasomal degradation of CDK1, which in turn enhances the phosphorylation of AR Ser81 by CDK1 and promotes the transcriptional activity of AR on downstream target genes. Inhibition of CDK1 not only eliminates the cancer-promoting effect of $\mathrm{ABCC} 5$ in prostate cancer but also increases the sensitivity of prostate cancer cells to enzalutamide.

\section{Supplementary Material}

Supplementary figures and tables. http://www.ijbs.com/v17p1613s1.pdf

\section{Acknowledgements}

\section{Funding}

This work was supported by the National Natural Science Foundation of China [81672546, 81772703, 81872083] and National Major New Drug Discovery Initiative' Fund [2017ZX09304030].

\section{Ethics Approval and Consent to Participate}

This study was approved by the ethics committee of the Peking University First Hospital, and written informed consent was obtained before any operation. Consent for publication. The authors confirmed that we obtained written consent from the patients to publish this manuscript.

\section{Data Availability Statement}

The datasets supporting the findings of this study are included within the article.

\section{Author Contributions}

GJJ and LQZ had designed the project. GJJ and $\mathrm{SMH}$ performed all the molecular biological experiments and data analysis and visualization. GJJ wrote the manuscript. $\mathrm{CH}, \mathrm{YQG}$, and XSL contributed to the language editing of this manuscript. All authors have read and approved the final manuscript.

\section{Competing Interests}

The authors have declared that no competing interest exists. 


\section{References}

1. Siegel RL, Miller KD, Jemal A. Cancer statistics, 2020. CA: a cancer journal for clinicians. 2020; 70: 7-30.

2. Fillon M. Rates of advanced prostate cancer continue to increase. CA: a cancer journal for clinicians. 2020; 70: 427-9.

3. Wilkins LJ, Tosoian JJ, Sundi D, Ross AE, Grimberg D, Klein EA, et al. Surgical management of high-risk, localized prostate cancer. Nat Rev Urol. 2020; 17: 679-90.

4. Powers E, Karachaliou GS, Kao C, Harrison MR, Hoimes CJ, George DJ, et al. Novel therapies are changing treatment paradigms in metastatic prostate cancer. J Hematol Oncol. 2020; 13: 144.

5. Connor MJ, Shah TT, Horan G, Bevan CL, Winkler M, Ahmed HU. Cytoreductive treatment strategies for de novo metastatic prostate cancer. Nat Rev Clin Oncol. 2020; 17: 168-82.

6. Coleman RE, Croucher PI, Padhani AR, Clezardin P, Chow E, Fallon M, et al. Bone metastases. Nat Rev Dis Primers. 2020; 6: 83.

7. Nuhn P, De Bono JS, Fizazi K, Freedland SJ, Grilli M, Kantoff PW, et al. Update on Systemic Prostate Cancer Therapies: Management of Metastatic Castration-resistant Prostate Cancer in the Era of Precision Oncology. European urology. 2019; 75: 88-99.

8. Fallowfield L, Payne H, Jenkins V. Patient-reported outcomes in metastatic castration-resistant prostate cancer. Nat Rev Clin Oncol. 2016; 13: 643-50.

9. Wilson BE, Hansen AR. Dual Checkpoint Blockade in Metastatic Castration-Resistant Prostate Cancer: Just a Gambit or Real CheckMate? Cancer cell. 2020; 38: 438-40.

10. Crabb SJ, Griffiths G, Marwood E, Dunkley D, Downs N, Martin K, et al. Pan-AKT Inhibitor Capivasertib With Docetaxel and Prednisolone in Metastatic Castration-Resistant Prostate Cancer: A Randomized, Placebo-Controlled Phase II Trial (ProCAID). Journal of clinical oncology: official journal of the American Society of Clinical Oncology. 2020: JCO2001576.

11. Kyriakopoulos CE, Heath EI, Ferrari A, Sperger JM, Singh A, Perlman $\mathrm{SB}$, et al. Exploring Spatial-Temporal Changes in (18)F-Sodium Fluoride PET/CT and Circulating Tumor Cells in Metastatic Castration-Resistant Prostate Cancer Treated With Enzalutamide. Journal of clinical oncology : official journal of the American Society of Clinical Oncology. 2020; 38: 3662-71.

12. Fizazi K, Kramer G, Eymard JC, Sternberg CN, de Bono J, Castellano D, et al. Quality of life in patients with metastatic prostate cancer following treatment with cabazitaxel versus abiraterone or enzalutamide (CARD): an analysis of a randomised, multicentre, open-label, phase 4 study. The Lancet Oncology. 2020; 21: 1513-25.

13. de Bono J, Mateo J, Fizazi K, Saad F, Shore N, Sandhu S, et al. Olaparib for Metastatic Castration-Resistant Prostate Cancer. The New England journal of medicine. 2020; 382: 2091-102.

14. Fizazi K, Shore N, Tammela TL, Ulys A, Vjaters E, Polyakov S, et al. Nonmetastatic, Castration-Resistant Prostate Cancer and Survival with Darolutamide. The New England journal of medicine. 2020; 383: 1040-9.

15. Hussain M, Mateo J, Fizazi K, Saad F, Shore N, Sandhu S, et al. Survival with Olaparib in Metastatic Castration-Resistant Prostate Cancer. The New England journal of medicine. 2020; 383: 2345-57.

16. Hou Y, Zhu Q, Li Z, Peng Y, Yu X, Yuan B, et al. The FOXM1-ABCC5 axis contributes to paclitaxel resistance in nasopharyngeal carcinoma cells. Cell death \& disease. 2017; 8: e2659.

17. Amponsah PS, Fan P, Bauer N, Zhao Z, Gladkich J, Fellenberg J, et al. microRNA-210 overexpression inhibits tumor growth and potentially reverses gemcitabine resistance in pancreatic cancer. Cancer letters. 2017; 388: 107-17.

18. Weaver DA, Crawford EL, Warner KA, Elkhairi F, Khuder SA, Willey JC. ABCC5, ERCC2, XPA and XRCC1 transcript abundance levels correlate with cisplatin chemoresistance in non-small cell lung cancer cell lines. Molecular cancer. 2005; 4: 18.

19. Pratt S, Shepard RL, Kandasamy RA, Johnston PA, Perry W, 3rd, Dantzig $\mathrm{AH}$. The multidrug resistance protein 5 (ABCC5) confers resistance to 5-fluorouracil and transports its monophosphorylated metabolites. Molecular cancer therapeutics. 2005; 4: 855-63.

20. Gharahkhani P, Fitzgerald RC, Vaughan TL, Palles C, Gockel I, Tomlinson I, et al. Genome-wide association studies in oesophageal adenocarcinoma and Barrett's oesophagus: a large-scale meta-analysis. The Lancet Oncology. 2016; 17: 1363-73.

21. Zhu Y, Yu F, Jiao Y, Feng J, Tang W, Yao H, et al. Reduced miR-128 in breast tumor-initiating cells induces chemotherapeutic resistance via Bmi-1 and ABCC5. Clinical cancer research : an official journal of the American Association for Cancer Research. 2011; 17: 7105-15.

22. Wu Q, Yang Z, Xia L, Nie Y, Wu K, Shi Y, et al. Methylation of miR-129-5p CpG island modulates multi-drug resistance in gastric cancer by targeting ABC transporters. Oncotarget. 2014; 5: 11552-63.
23. Wang Z, Yang J, Xu G, Wang W, Liu C, Yang H, et al. Targeting miR-381-NEFL axis sensitizes glioblastoma cells to temozolomide by regulating stemness factors and multidrug resistance factors. Oncotarget. 2015; 6: 3147-64.

24. Li B, Severson E, Pignon JC, Zhao H, Li T, Novak J, et al. Comprehensive analyses of tumor immunity: implications for cancer immunotherapy. Genome Biol. 2016; 17: 174.

25. Li T, Fan J, Wang B, Traugh N, Chen Q, Liu JS, et al. TIMER: A Web Server for Comprehensive Analysis of Tumor-Infiltrating Immune Cells. Cancer research. 2017; 77: e108-e10.

26. Li T, Fu J, Zeng Z, Cohen D, Li J, Chen Q, et al. TIMER2.0 for analysis of tumor-infiltrating immune cells. Nucleic Acids Res. 2020; 48: W509-W14.

27. Langfelder $\mathrm{P}$, Horvath $\mathrm{S}$. WGCNA: an $\mathrm{R}$ package for weighted correlation network analysis. BMC Bioinformatics. 2008; 9: 559.

28. Wang $\mathrm{XQ}, \mathrm{Lo} \mathrm{CM}$, Chen L, Ngan ES, $\mathrm{Xu} A$, Poon RY. CDK1-PDK1-PI3K/Akt signaling pathway regulates embryonic and induced pluripotency. Cell Death Differ. 2017; 24: 38-48.

29. Kaibori Y, Saito Y, Nakayama Y. EphA2 phosphorylation at Ser897 by the Cdk1/MEK/ERK/RSK pathway regulates M-phase progression via maintenance of cortical rigidity. FASEB journal : official publication of the Federation of American Societies for Experimental Biology. 2019; 33: 5334-49.

30. Litviakov NV, Cherdyntseva NV, Tsyganov MM, Slonimskaya EM, Ibragimova MK, Kazantseva PV, et al. Deletions of multidrug resistance gene loci in breast cancer leads to the down-regulation of its expression and predict tumor response to neoadjuvant chemotherapy. Oncotarget. 2016; 7: 7829-41.

31. Nies AT, Magdy T, Schwab M, Zanger UM. Role of ABC transporters in fluoropyrimidine-based chemotherapy response. Advances in cancer research. 2015; 125: 217-43.

32. Hagmann W, Jesnowski R, Lohr JM. Interdependence of gemcitabine treatment, transporter expression, and resistance in human pancreatic carcinoma cells. Neoplasia (New York, NY). 2010; 12: 740-7.

33. Zhang W, Shannon WD, Duncan J, Scheffer GL, Scheper RJ, McLeod HL. Expression of drug pathway proteins is independent of tumour type. The Journal of pathology. 2006; 209: 213-9.

34. Karatas OF, Guzel E, Duz MB, Ittmann M, Ozen M. The role of ATP-binding cassette transporter genes in the progression of prostate cancer. The Prostate. 2016; 76: 434-44.

35. Zhang H, Lian Z, Sun G, Liu R, Xu Y. Loss of miR-516a-3p mediates upregulation of ABCC5 in prostate cancer and drives its progression. Onco Targets Ther. 2018; 11: 3853-67.

36. Park S, Shimizu C, Shimoyama T, Takeda M, Ando M, Kohno T, et al. Gene expression profiling of ATP-binding cassette (ABC) transporters as a predictor of the pathologic response to neoadjuvant chemotherapy in breast cancer patients. Breast cancer research and treatment. 2006; 99: 9-17.

37. Wielinga P, Hooijberg JH, Gunnarsdottir S, Kathmann I, Reid G, Zelcer $\mathrm{N}$, et al. The human multidrug resistance protein MRP5 transports folates and can mediate cellular resistance against antifolates. Cancer research. 2005; 65: 4425-30.

38. Gazzaniga P, Gradilone A, Petracca A, Nicolazzo C, Raimondi C, Iacovelli $\mathrm{R}$, et al. Molecular markers in circulating tumour cells from metastatic colorectal cancer patients. Journal of cellular and molecular medicine. 2010; 14: 2073-7.

39. Denisov EV, Litviakov NV, Zavyalova MV, Perelmuter VM, Vtorushin $\mathrm{SV}$, Tsyganov MM, et al. Intratumoral morphological heterogeneity of breast cancer: neoadjuvant chemotherapy efficiency and multidrug resistance gene expression. Scientific reports. 2014; 4: 4709.

40. Wang JQ, Yang Y, Cai CY, Teng QX, Cui Q, Lin J, et al. Multidrug resistance proteins (MRPs): Structure, function and the overcoming of cancer multidrug resistance. Drug resistance updates : reviews and commentaries in antimicrobial and anticancer chemotherapy. 2021; 54: 100743.

41. McAleer MA, Breen MA, White NL, Matthews N. pABC11 (also known as MOAT-C and MRP5), a member of the ABC family of proteins, has anion transporter activity but does not confer multidrug resistance when overexpressed in human embryonic kidney 293 cells. The Journal of biological chemistry. 1999; 274: 23541-8.

42. Wijnholds J, Mol CA, van Deemter L, de Haas M, Scheffer GL, Baas F, et al. Multidrug-resistance protein 5 is a multispecific organic anion transporter able to transport nucleotide analogs. Proceedings of the National Academy of Sciences of the United States of America. 2000; 97: 7476-81.

43. Lockhart AC, Tirona RG, Kim RB. Pharmacogenetics of ATP-binding cassette transporters in cancer and chemotherapy. Molecular cancer therapeutics. 2003; 2: 685-98. 
44. Pan ST, Li ZL, He ZX, Qiu JX, Zhou SF. Molecular mechanisms for tumour resistance to chemotherapy. Clinical and experimental pharmacology \& physiology. 2016; 43: 723-37.

45. Guan X, Sun D, Lu E, Urrutia JA, Reiter RE, Rettig M, et al. Copy Number Loss of 17q22 Is Associated with Enzalutamide Resistance and Poor Prognosis in Metastatic Castration-Resistant Prostate Cancer. Clinical cancer research : an official journal of the American Association for Cancer Research. 2020; 26: 4616-24.

46. Liu P, Kao TP, Huang H. CDK1 promotes cell proliferation and survival via phosphorylation and inhibition of FOXO1 transcription factor. Oncogene. 2008; 27: 4733-44.

47. Liu X, Gao Y, Ye H, Gerrin S, Ma F, Wu Y, et al. Positive feedback loop mediated by protein phosphatase 1alpha mobilization of P-TEFb and basal CDK1 drives androgen receptor in prostate cancer. Nucleic Acids Res. 2017; 45: 3738-51.

48. Tsaur I, Makarevic J, Hudak L, Juengel E, Kurosch M, Wiesner C, et al. The cdk1-cyclin B complex is involved in everolimus triggered resistance in the PC3 prostate cancer cell line. Cancer letters. 2011; 313: 84-90.

49. Chen S, Xu Y, Yuan X, Bubley GJ, Balk SP. Androgen receptor phosphorylation and stabilization in prostate cancer by cyclin-dependent kinase 1. Proceedings of the National Academy of Sciences of the United States of America. 2006; 103: 15969-74.

50. Zhang C, Elkahloun AG, Robertson M, Gills JJ, Tsurutani J, Shih JH, et al. Loss of cytoplasmic CDK1 predicts poor survival in human lung cancer and confers chemotherapeutic resistance. PloS one. 2011; 6: e23849. 